\title{
Analysis and validation of correlation curves between sclerometry and compression tests for the evaluation of compressive strength of concretes
}

\section{Análise e validação de curvas de correlação entre a esclerometria e ensaios de compressão para avaliação da resistência à compressão de concretos}
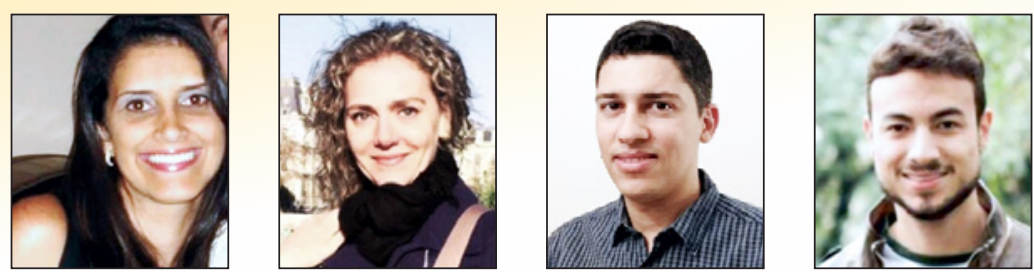

T. M. OLIVEIRA a thaismayra@yahoo.com.br

M. C. R. FARAGE faragebr@gmail.com

L. GOLIATT leonardo.goliatt@engenharia.ufjf.br

T. C. BITTENCOURT a thiago.bittencourt@engenharia.ufjf.br

\section{Abstract}

The present work consists in obtaining correlation curves between compressive tests and sclerometry tests from an extensive experimental program comprising 630 concrete cylindrical samples cast in laboratory, employing materials and methods available in Brazil. The concretes, with compressive strengths ranging from 20 MPa to $50 \sim \mathrm{MPa}$, were submitted to sclerometry tests and mechanical compressive tests. The results were used to obtain correlation curves. which were adopted in measurements of structural elements molded in a construction site, indicating that the curves provided by the equipment manufacturer are not suitable for the material in study. This is the first stage of a broader study aiming to contribute to the improvement of the Brazilian code regulating the application of the sclerometry to obtain concrete compressive strength.

Keywords: concrete, sclerometry, compressive strength, correlation curves.

\section{Resumo}

Este trabalho consiste na obtenção de curvas de correlação entre ensaios mecânicos e testes de esclerometria a partir de um programa experimental extenso realizado em 630 corpos de prova de concreto. empregando materiais e métodos disponíveis no Brasil. Os concretos, com resistências a compressão variando de $20 \mathrm{MPa}$ a $50 \mathrm{MPa}$, foram submetidos a ensaios de esclerometria e a testes mecânicos de compressão, cujos resultados foram empregados na obtenção de curvas de correlação. Tais curvas foram adotadas em medições de elementos estruturais moldados em um canteiro de obras, indicando que as curvas fornecidas pelo fabricante do equipamento não são adequadas para o material em estudo. Trata-se da primeira etapa de um amplo estudo visando contribuir para o aprimoramento das normas brasileiras que estabelecem os procedimentos para a aplicação da esclerometria para medir a resistência a compressão de concreto.

Palavras-chave: concreto, esclerometria, resistência a compressão, curva de correlação.

\footnotetext{
Programa de Pós-Graduação em Engenharia Civil, Departamento de Construção Civil. Faculdade de Engenharia. Universidade Federal de Juiz de Fora. Juiz de Fora, MG, Brasil, Programa de Pós-Graduação em Modelagem Computacional, Departamento de Mecânica Aplicada e Computacional, Faculdade de Engenharia, Universidade Federal de Juiz de Fora, Juiz de Fora, MG, Brasil.
}

Received: 04 Apr 2017 • Accepted: 05 Apr 2018 • Available Online: 


\section{Introduction}

Non-Destructive Tests (NDT) are considered as important tools for the structural and quality evaluations of construction elements. Concerning mechanical properties of concrete structures in service conditions it is quite convenient to adopt in situ techniques that do not demand carroting, both for economical and safety reasons, since extracting samples from structural elements may cause deterioration and/or affect the serviceability or the entire structure. These facts certainly contributed to spreading the application of non-destructive techniques, such as the sclerometry, all over the world (Breysse et al [11], Fan et al [13], Fort et al [14], Sardar et al [18]).

The sclerometer measures essentially the hardness of the surface. The method is based on the rebound principle, in which an elongated spring, upon returning to the equilibrium position, causes a hammer with a certain mass to move colliding against the surface of an object with a much larger mass. After the impact the deformation of the spring is measured through extensometers, present inside the apparatus. The measured deformation provides the sclerometric index, which is shown in the apparatus. With this index empirical correlations can be made with compression tests (Ayman and Mohamed [10]. Malhotra and Carino [16]). The device used herein (Figure 1(a)) was designed by the Swiss engineer Ernst Schmidt - so it is also known as Schmidt sclerometer. Nowadays its use is very widespread: it is estimated that up to 1986 had already been sold around fifty thousand sclerometers (Malhotra and Carino [16]). Each type of sclerometer provides a specific set of curves correlating sclerometry indexes to compressive strength values obtained via mechanical tests (Malhotra and Carino [16]). Figure 1(b) shows the correlation curves provided by the manufacturers of the employed sclerometer model N/NR where three shot directions are considered: horizontal, vertical downwards and vertical upwards. As one can see by looking at the curves, the results are considerably affected by the shot direction. However, it is well known that those curves do not fit a wide variety of concretes but are restricted to specific materials and test conditions. This fact is a handicap for the application of this procedure in a wide manner.

The present study consists of an extensive experimental program concerning two steps: firstly, 630 cylindrical concrete samples were fabricated and subjected to sclerometer and mechanical tests in order to obtain the correlation curves; secondly, measurements were performed in concrete structural members in a construction site, so as to reproduce a practical situation of material's verification and control under in situ conditions, and to compare the results obtained from the correlation curves to those measured via mechanical tests on cylindrical concrete samples for technological control purposes.

The aim of this work is contributing for the reliable application of sclerometry devices in Brazil, since the correlation curves provided by the commonly adopted equipments may not suit the local materials. Further, the related Brazilian Standard (ABNT NBR 7584:2012 - Concreto endurecido — Avaliação da dureza superficial pelo esclerômetro de reflexão - Método de ensaio) (ABNT [8]) does not provide detailed information concerning the assessment and application of adequate correlation curves, which limits the application of this technique for practical purposes.

\section{Experimental program}

Firstly, an extensive experimental study was developed so as to generate sufficient and reliable data. The experimental program comprised 7 classes of concrete samples identified as CXX, where $\mathrm{XX}$ indicates the compressive strength value specified in the pro-

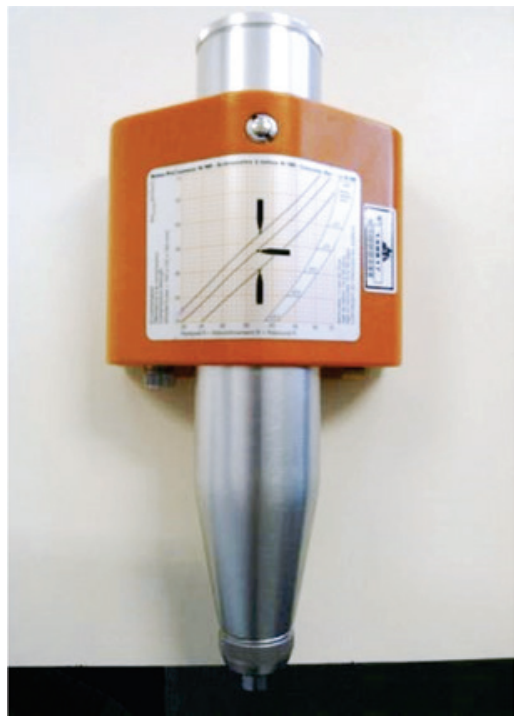

a

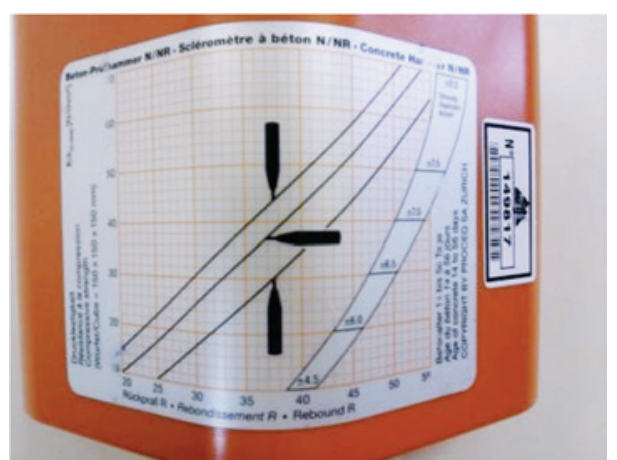

b

Figure 1

(a) Sclerometer model N/NR; (b) Correlation curves provided by the manufacturer considering 3 shot application directions 


\section{Table 1}

Compositions (given in mass) adopted for the seven concrete sets

\begin{tabular}{|c|c|c|c|c|c|c|c|c|}
\hline Concrete & $\begin{array}{c}\mathbf{f}_{\text {ck }} \\
(\mathbf{M P a})\end{array}$ & $\begin{array}{c}\text { Artificial } \\
\text { sand }\end{array}$ & $\begin{array}{c}\text { Natural } \\
\text { sand }\end{array}$ & B0 & B1 & w/c & $\begin{array}{c}\text { Add. } \\
\%\end{array}$ & $\begin{array}{c}\text { Cement kg/ } \\
\mathbf{m}^{\mathbf{3}}\end{array}$ \\
\hline C20 & 20 & 0.924 & 2.772 & 1.200 & 2.800 & 0.65 & 0.65 & 260 \\
\hline C25 & 25 & 0.843 & 2.528 & 1.117 & 2.607 & 0.60 & 0.65 & 280 \\
\hline C30 & 30 & 0.700 & 2.099 & 0.971 & 2.265 & 0.55 & 0.65 & 320 \\
\hline C35 & 35 & 0.644 & 1.933 & 0.914 & 2.133 & 0.52 & 0.65 & 340 \\
\hline C40 & 40 & 0.621 & 1.864 & 0.891 & 2.078 & 0.50 & 0.65 & 350 \\
\hline C45 & 45 & 0.611 & 1.832 & 0.880 & 2.052 & 0.45 & 0.65 & 360 \\
\hline C50 & 50 & 0.436 & 1.307 & 0.701 & 1.635 & 0.38 & 0.65 & 450 \\
\hline
\end{tabular}

Table 2

Aggregate's grading

\begin{tabular}{|c|c|c|c|c|}
\hline \multicolumn{5}{|c|}{ Grading (ABNT [2]) } \\
\hline \multirow{2}{*}{ Diameter (mm) } & \multicolumn{4}{|c|}{$\%$ retained (accumulated) } \\
\hline & BO & B1 & NS & AS \\
\hline 25 & - & - & - & - \\
\hline 19 & - & 14 & - & - \\
\hline 12.5 & - & 77 & - & - \\
\hline 9.5 & 6 & 98 & - & - \\
\hline 6.3 & 60 & 99 & 1 & - \\
\hline 4.8 & 89 & 99 & 1 & 1 \\
\hline 2.4 & 99 & 99 & 4 & 22 \\
\hline 1.2 & 99 & 99 & 21 & 42 \\
\hline 0.6 & 99 & 99 & 58 & 53 \\
\hline 0.3 & 99 & 99 & 89 & 62 \\
\hline 0.15 & 99 & 99 & 98 & 76 \\
\hline Bottom & 100 & 100 & 100 & 100 \\
\hline Specific mass $\left(\mathrm{g} / \mathrm{cm}^{3}\right)$ (ABNT [4]) (ABNT [5]) & 2.76 & 2.85 & 2.6 & 2.725 \\
\hline Unity mass $\left(\mathrm{g} / \mathrm{cm}^{3}\right)($ ABNT $[1])$ & 1.4 & 1.49 & 1.44 & 1.72 \\
\hline Amount of fine materials (\%) (ABNT [3]) & 0.685 & 0.945 & 2.25 & 20.8 \\
\hline
\end{tabular}

portioning specifications. Table 1 shows the mixture's proportions, pre-defined for compressive strengths ranging from $20 \mathrm{MPa}$ to 50 MPa. All mixtures adopted cement type CPIIIE40, natural and artificial sand and polifunctional plastificant additive. Water/cement ratio ranged from 0.38 to 0.65 .

All materials adopted in the concretes fabrication were characterized according to specific standardized tests - grading analysis, specific mass, amount of fine grains - for the sake of reproducibility. Mix proportions, as well as water/cement ratios, were defined in terms of aggregates grading and the amount of fine grains (diameter $<0.075 \mathrm{~mm}$ ) given in Table 2 (Mehta and Monteiro [17]). Following the pre-definition of mixture proportions, 11 cylindrical samples $(100 \times 200 \mathrm{~mm}$ according to NBR 5738 (ABNT [6])) per concrete class were molded aiming to verify the compressive strength values. Then 90 concrete cylindrical samples $(100 \times 200 \mathrm{~mm})$ per strength class, totalizing 630 specimens, were fabricated in order to obtain enough data for the analysis. Figure 2(a) illustrates the specimens' fabrication. The cylindrical samples were kept in molds for about 72 hours under controlled moisture conditions. then they were unmolded and kept under plain water as shown in Figure 2(b). At the age of 27 days, the specimens were taken off the water for surface regularization, as illustrated in Figure 2(c), and subjected to sclerometry and compressive tests 28 days after molding.

The Brazilian Standard NBR 7584 (ABNT [12]) states that a concrete specimen must be subjected to 16 impact points on its surface, defined as the nodes of a $3 \times 3$ mesh like the one shown in Figure 3(a). However, the present work adopted 4 impact points, as indicated in Figure 3(b), due to the small dimensions of the concrete cylinders in study.

For each mixture, 90 specimens were grouped into 3 sets of 30 samples. Each set was subjected to sclerometry measurements under one of the three shot directions indicated in Figure 4, identified herein as: Position $1(\rightarrow)$ (Figure 4(a)); Position $2(\downarrow)$ (Figure 4(b)) and Position $3-(\uparrow)$ (Figure 4(c)). Mechanical uniaxial tests were also performed according to Brazilian Standard NBR 5739:2007 (ABNT [7]) for the evaluation of the compression strength $\left(f_{c}\right)$, so as to provide sufficient data for analysis and validation purposes. 


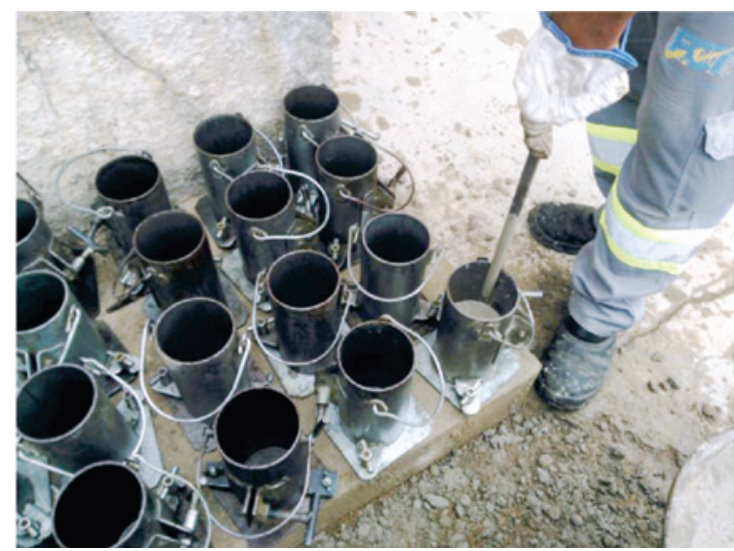

a

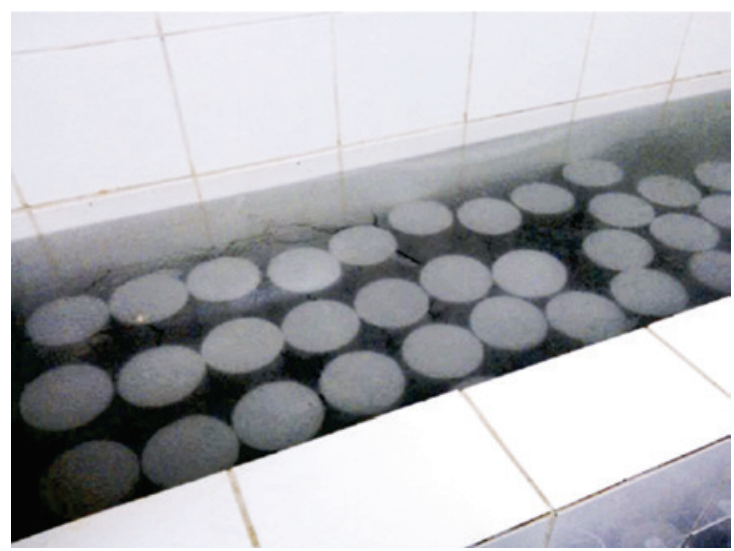

b

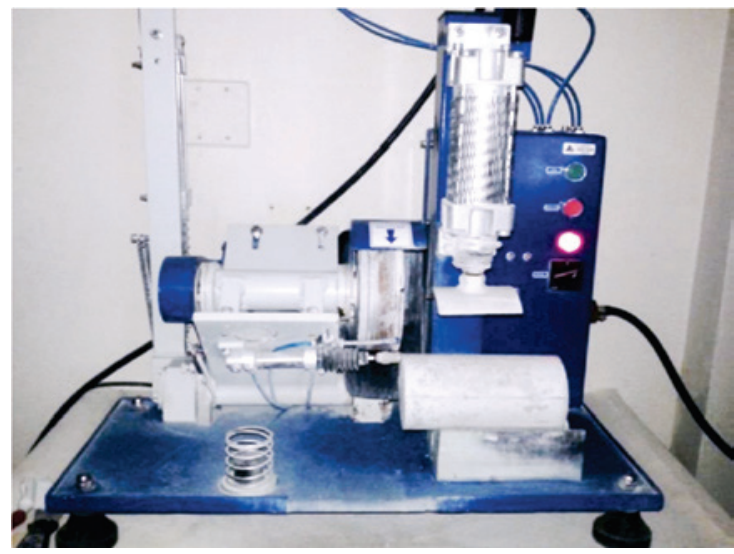

C

\section{Figure 2}

Fabrication of the concrete's specimens (a) water curing (b) and regularization of the top surfaces

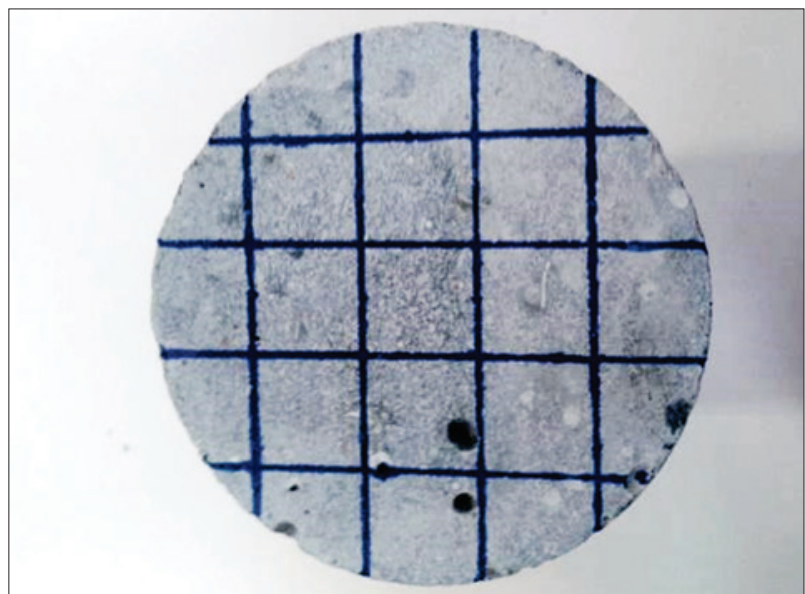

a

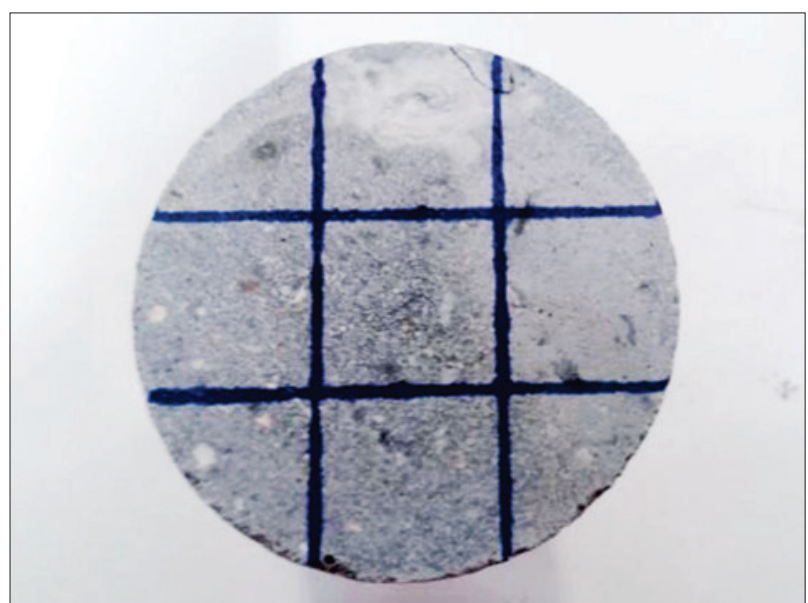

b

\section{Figure 3}

(a) Mesh with 16 impact points as stated by NBR 7584:2012 (ABNT [12]) and (b) Mesh with 4 impact points, as adopted in the present study 


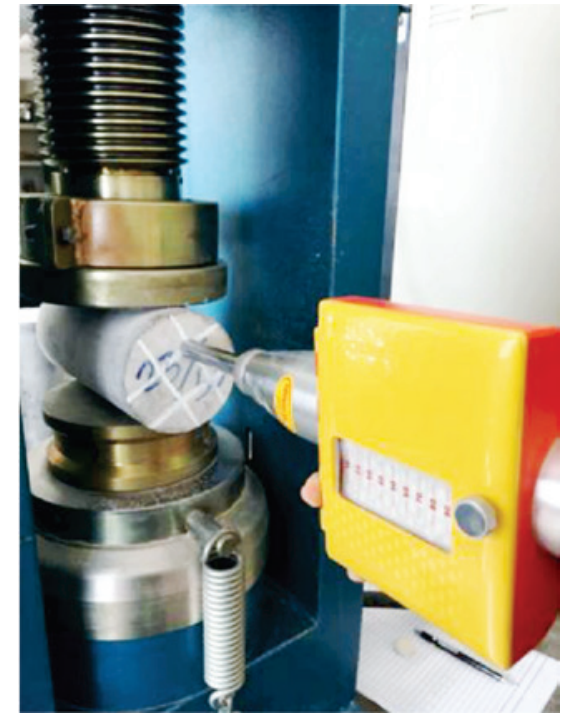

a

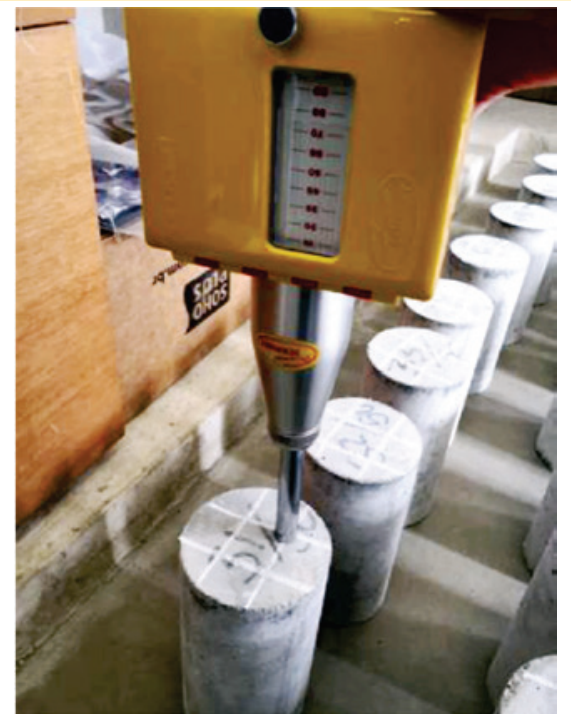

b

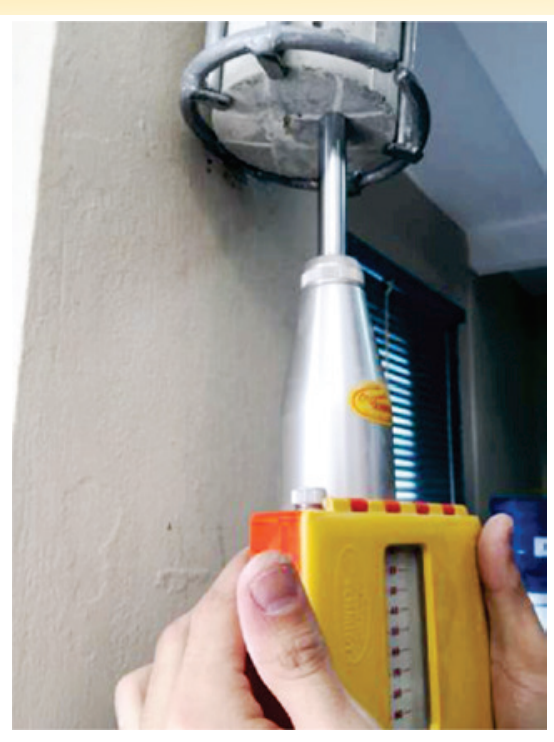

c

Figure 4

Sclerometry test performed in three positions: (a) Position $1(\rightarrow)$ (b) vertical downwards (Position 2) and (c) vertical upwards (Position 3)

\section{Table 3}

Experimental data obtained for C20 concrete - Set 1

\begin{tabular}{|c|c|c|c|c|c|c|c|}
\hline Item & Position & 11 & 12 & 13 & 14 & Ia & $f_{c}(M P a)$ \\
\hline 1 & $\downarrow$ & 12 & 14 & 12 & 12 & 12.5 & 18.47 \\
\hline 2 & $\downarrow$ & 14 & 14 & 14 & 14 & 14.0 & 20.66 \\
\hline 3 & $\downarrow$ & 14 & 14 & 16 & 16 & 15.0 & 19.62 \\
\hline 4 & $\downarrow$ & 16 & 14 & 16 & 16 & 15.5 & 20.54 \\
\hline $5 *$ & $\downarrow$ & 15 & 15 & 14.5 & 14.5 & 14.8 & 20.66 \\
\hline 6* & $\downarrow$ & 13 & 13 & 14 & 14 & 13.5 & 21.16 \\
\hline 7 & $\downarrow$ & 13 & 13 & 13 & 13 & 13.0 & 21.15 \\
\hline 8 & $\downarrow$ & 14 & 12 & 12 & 13 & 12.8 & 23.81 \\
\hline 9 & $\downarrow$ & 13.5 & 13 & 13 & 13 & 13.1 & 21.21 \\
\hline 10 & $\downarrow$ & 18 & 13 & 13 & 13 & 13.0 & 25.64 \\
\hline 11 & $\uparrow$ & 16 & 16 & 16 & 16 & 16.0 & 22.68 \\
\hline $12^{*}$ & $\uparrow$ & 16 & 16 & 15 & 15 & 15.5 & 20.82 \\
\hline 13 & $\uparrow$ & 16 & 15 & 15 & 17 & 15.8 & 23.53 \\
\hline 14 & $\uparrow$ & 15 & 16 & 15 & 16 & 15.5 & 23.52 \\
\hline 15 & $\uparrow$ & 16 & 16 & 16 & 16 & 16.0 & 25.68 \\
\hline 16 & $\uparrow$ & 16 & 16 & 16 & 16 & 16.0 & 21.59 \\
\hline 17 & $\uparrow$ & 17 & 16 & 16 & 16 & 16.3 & 18.91 \\
\hline 18 & $\uparrow$ & 21 & 17 & 16 & 16 & 16.3 & 21.39 \\
\hline 19 & $\uparrow$ & 15 & 15 & 14 & 15 & 14.8 & 22.61 \\
\hline 20 & $\uparrow$ & 15 & 15 & 15 & 15 & 15.0 & 20.79 \\
\hline 21 & $\rightarrow$ & 14 & 14 & 14 & 14 & 14.0 & 22.04 \\
\hline 22 & $\rightarrow$ & 15 & 14 & 14 & 15 & 14.5 & 20.41 \\
\hline 23 & $\rightarrow$ & 14 & 14 & 14 & 15 & 14.3 & 18.47 \\
\hline 24 & $\rightarrow$ & 13 & 14 & 14 & 13 & 13.5 & 23.94 \\
\hline 25 & $\rightarrow$ & 16 & 16 & 16 & 15 & 15.8 & 24.23 \\
\hline 26 & $\rightarrow$ & 17 & 16 & 16 & 16 & 16.3 & 26.32 \\
\hline 27 & $\rightarrow$ & 14 & 14 & 14 & 14 & 14.0 & 17.32 \\
\hline 28 & $\rightarrow$ & 13 & 14 & 14 & 14 & 13.8 & 19.01 \\
\hline 29 & $\rightarrow$ & 13.5 & 13.5 & 13.5 & 14 & 13.6 & 14.69 \\
\hline 30 & $\rightarrow$ & 14 & 14 & 14 & 14 & 14.0 & 19.20 \\
\hline
\end{tabular}


Information on the experimental measurements obtained from all tested samples are presented in Tables 3 to 23, where: 11, 12, 13 and 14 are the sclerometer index readings, obtained as described in section $1, I_{a}$ is the average index value, Position indicates the shot direction, fc is the compressive strength.

\section{Results and discussions}

\subsection{I $\times f_{c}$ correlation curves}

The database was treated using tests for Normal and Student distribution and Pearson correlation coefficient and Kolmogorov-Smirnov two sample tests were used as a measure of the quality of the correlation (Figueiredo Filho and Silva [15]). The analysis and validation of the curves were done with the help of the python programming language, using the numpy, scipy and pandas packages for the numerical and statistical processing. Detailed information concerning the database and correlation analysis is available in Botelho et al [11].
According to Figure 5, in all the ranges of $\mathrm{fc}$ it is observed that the distribution of the values in Position $3(\uparrow)$ presents higher sclerometer indices than in Position $2(\downarrow)$. Observing the diagram of Figure 5 , it can be seen that the mean values of the sclerometer index (I) increase with the fc ranges, suggesting a linear behavior. In addition to that, the three directions present different mean values, Position $3(\uparrow)$ having the highest one, followed by Position $1(\rightarrow)$ and Position $2(\downarrow)$.

The linear regression models of the form $\mathrm{f}_{c}=\mathrm{al}+\mathrm{b}$ for the sclerometer index as a function of each of the shot directions are presented in Table 24. The MSE column indicates the mean square error, the $\mathrm{R}$ column indicates the Pearson correlation coefficient and the $p$ column indicates the $p$-value associated with the regression model. The same data is plot on Figure 6 , which allows the graphical comparison of the linear regression models for the sclerometer index as a function of the shot directions. One can compare the trend lines obtained for the three adopted directions. which respect a certain distance from each other.

\section{Table 4}

Experimental data obtained for $\mathrm{C} 20$ concrete samples - Set 2

\begin{tabular}{|c|c|c|c|c|c|c|c|}
\hline Item & Position & 11 & 12 & 13 & 14 & Ia & $f_{c}(M P a)$ \\
\hline 31 & $\downarrow$ & 14 & 14 & 13 & 13 & 13.5 & 17.67 \\
\hline 32 & $\downarrow$ & 14 & 13 & 13 & 14 & 13.5 & 17.65 \\
\hline 33 & $\downarrow$ & 12.5 & 12.5 & 13 & 13 & 12.8 & 16.04 \\
\hline 34 & $\downarrow$ & 12.5 & 12.5 & 12.5 & 12 & 12.4 & 17.55 \\
\hline 35 & $\downarrow$ & 12.5 & 12.5 & 12 & 12 & 12.3 & 18.23 \\
\hline 36 & $\downarrow$ & $10 * *$ & 14.5 & 14.5 & 13.5 & 14.2 & 18.51 \\
\hline 37 & $\downarrow$ & 13 & 13 & 13 & 13 & 13.0 & 17.72 \\
\hline 38 & $\downarrow$ & 13 & 13 & 13 & $17^{* *}$ & 13.0 & 19.12 \\
\hline 39 & $\downarrow$ & 13.5 & 12.5 & 13.5 & 13.5 & 13.3 & 18.92 \\
\hline 40 & $\downarrow$ & 14 & 14 & 13 & 13 & 13.5 & 19.30 \\
\hline 41 & $\uparrow$ & 17 & 16 & 16 & 17 & 16.5 & 17.14 \\
\hline 42 & $\uparrow$ & 16.5 & 15.5 & 16.5 & $10 * *$ & 16.2 & 17.83 \\
\hline 43 & $\uparrow$ & 16 & 16 & 16 & 16 & 16.0 & 17.49 \\
\hline 44 & $\uparrow$ & 16 & 16 & 16 & 16 & 16.0 & 17.57 \\
\hline 45 & $\uparrow$ & 16 & 16 & 16 & 15 & 15.8 & 17.77 \\
\hline 46 & $\uparrow$ & 15.5 & 16 & 16 & 16 & 15.9 & 18.25 \\
\hline 47 & $\uparrow$ & 15 & 15.5 & 15.5 & 15.5 & 15.4 & 20.12 \\
\hline 48 & $\uparrow$ & 16 & 16 & 16 & 16 & 16.0 & 19.40 \\
\hline 49 & $\uparrow$ & 16 & 16 & 16 & 16.5 & 16.1 & 20.22 \\
\hline 50 & $\uparrow$ & 16 & 16 & 15 & 15 & 15.5 & 19.43 \\
\hline 51 & $\rightarrow$ & 14 & 14 & 14 & 14.5 & 14.1 & 18.03 \\
\hline 52 & $\rightarrow$ & 16 & 15 & 15 & 16 & 15.5 & 19.20 \\
\hline 53 & $\rightarrow$ & 13.5 & 14 & 15 & $10 * *$ & 14.2 & 18.49 \\
\hline 54 & $\rightarrow$ & 14 & 15 & 15 & 15 & 14.8 & 17.94 \\
\hline 55 & $\rightarrow$ & 14 & 14 & 13 & 14 & 13.8 & 18.72 \\
\hline 56 & $\rightarrow$ & 13 & 13.5 & 14.5 & $20 * *$ & 13.7 & 17.84 \\
\hline 57 & $\rightarrow$ & 14 & 14 & 13 & 13 & 13.5 & 18.89 \\
\hline 58 & $\rightarrow$ & 13.5 & 13.5 & 12.5 & 12.5 & 13.0 & 18.00 \\
\hline 59 & $\rightarrow$ & 14 & 14 & 14 & 14 & 14.0 & 19.63 \\
\hline 60 & $\rightarrow$ & 14 & 14 & 14 & 14 & 14.0 & 20.09 \\
\hline
\end{tabular}




\section{Table 5}

Experimental data obtained for C20 concrete samples - Set 3

\begin{tabular}{|c|c|c|c|c|c|c|c|}
\hline Item & Position & 11 & 12 & 13 & 14 & la & $f_{c}(M P a)$ \\
\hline 61 & $\downarrow$ & 14 & 14 & 14 & 14 & 14.0 & 18.95 \\
\hline 62 & $\downarrow$ & 15 & 15 & 15 & 15 & 15.0 & 19.40 \\
\hline 63 & $\downarrow$ & 15 & 15 & 15 & 15 & 15.0 & 19.51 \\
\hline 64 & $\downarrow$ & 14 & 14 & 15 & 15 & 14.5 & 18.13 \\
\hline 65 & $\downarrow$ & 16 & 15 & 15 & 16 & 15.5 & 19.38 \\
\hline 66 & $\downarrow$ & 15 & 15 & 15 & 15 & 15.0 & 19.96 \\
\hline 67 & $\downarrow$ & 14 & 14 & 14 & 14 & 14.0 & 19.63 \\
\hline 68 & $\downarrow$ & 16 & 16 & 16 & 16 & 16.0 & 19.48 \\
\hline 69 & $\downarrow$ & 17 & 17 & 16 & 16 & 16.5 & 20.45 \\
\hline 70 & $\downarrow$ & 15 & 15 & 14 & 14 & 14.5 & 17.80 \\
\hline 71 & $\uparrow$ & 16 & 17 & 16 & 17 & 16.5 & 19.51 \\
\hline 72 & $\uparrow$ & 16 & 16 & 16 & 16 & 16.0 & 19.57 \\
\hline 73 & $\uparrow$ & 16.5 & 16 & 15 & 15.5 & 15.8 & 18.26 \\
\hline 74 & $\uparrow$ & 15.5 & 15.5 & 14 & 14.5 & 14.9 & 18.54 \\
\hline 75 & $\uparrow$ & 16.5 & 16 & 16 & $10^{* *}$ & 16.2 & 19.46 \\
\hline 76 & $\uparrow$ & 17.5 & 17 & 16 & 16.5 & 16.8 & 20.35 \\
\hline 77 & $\uparrow$ & 16 & 16 & 15 & 15 & 15.5 & 18.87 \\
\hline 78 & $\uparrow$ & 17 & 17.5 & 16 & 16.5 & 16.8 & 19.20 \\
\hline 79 & $\uparrow$ & $12^{* *}$ & 16 & 16 & 16.5 & 16.2 & 19.33 \\
\hline 80 & $\uparrow$ & 15 & 15 & 15 & 15 & 15.0 & 18.16 \\
\hline 81 & $\rightarrow$ & 14 & 14 & 14 & 14 & 14.0 & 18.13 \\
\hline 82 & $\rightarrow$ & 17 & 16 & 16 & 17 & 16.5 & 20.32 \\
\hline 83 & $\rightarrow$ & 15.5 & 15.5 & 15.5 & 15.5 & 15.5 & 18.44 \\
\hline 84 & $\rightarrow$ & 17 & 17 & 16 & 16 & 16.5 & 18.18 \\
\hline 85 & $\rightarrow$ & 14 & 14 & $19^{* *}$ & 14.5 & 14.2 & 18.28 \\
\hline 86 & $\rightarrow$ & 14 & 14 & 14 & 14 & 14.0 & 20.27 \\
\hline 87 & $\rightarrow$ & 16 & 16 & 15 & 15 & 15.5 & 19.40 \\
\hline 88 & $\rightarrow$ & 15.5 & 15.5 & 15.5 & 15.5 & 15.5 & 19.12 \\
\hline 89 & $\rightarrow$ & 15 & 15 & 15 & 15 & 15.0 & 18.67 \\
\hline 90 & $\rightarrow$ & 14 & 14.5 & 13 & 13.5 & 13.8 & 19.89 \\
\hline
\end{tabular}


Table 6

Experimental data obtained for C25 concrete samples - Set 1

\begin{tabular}{|c|c|c|c|c|c|c|c|}
\hline Item & Position & 11 & 12 & 13 & 14 & la & $f_{c}(M P a)$ \\
\hline 1 & $\downarrow$ & 17 & 17 & 17 & 17 & 17.0 & 23.15 \\
\hline 2 & $\downarrow$ & 17 & 17 & 16 & 16 & 16.5 & 25.38 \\
\hline 3 & $\downarrow$ & 16 & 16 & 16 & 16 & 16.0 & 27.81 \\
\hline 4 & $\downarrow$ & 18 & 18 & 18 & 18 & 18.0 & 24.87 \\
\hline 5* & $\downarrow$ & 19 & 19 & 19 & 19 & 19.0 & 24.87 \\
\hline 6 & $\downarrow$ & 16 & 15.5 & 16 & 16 & 15.9 & 26.17 \\
\hline 7 & $\downarrow$ & 15 & 15 & 15 & 15 & 15.0 & 23.40 \\
\hline 8 & $\downarrow$ & 16 & 16 & 17 & 17 & 16.5 & 22.41 \\
\hline 9 & $\downarrow$ & 16.5 & 16.5 & 16.5 & 17 & 16.6 & 26.10 \\
\hline 10 & $\downarrow$ & 15 & 15 & 15 & 15 & 15.0 & 24.64 \\
\hline 11 & $\uparrow$ & 21 & 21 & 21 & 21 & 21.0 & 22.84 \\
\hline 12 & $\uparrow$ & 21 & 21 & 21 & 21 & 21.0 & 23.35 \\
\hline 13 & $\uparrow$ & 19 & 19 & 19 & 19 & 19.0 & 22.63 \\
\hline 14 & $\uparrow$ & 19 & 19 & 19 & 19 & 19.0 & 24.09 \\
\hline 15 & $\uparrow$ & 19 & 19 & 19 & 19 & 19.0 & 26.47 \\
\hline 16 & $\uparrow$ & 19.5 & 19.5 & 19.5 & 20 & 19.6 & 22.51 \\
\hline 17 & $\uparrow$ & 22 & 22 & 22.5 & $14^{* *}$ & 22.2 & 24.04 \\
\hline 18 & $\uparrow$ & 22.5 & 22.5 & 22.5 & 23 & 22.6 & 25.17 \\
\hline 19 & $\uparrow$ & 22 & 22 & 22 & 22 & 22.0 & 22.70 \\
\hline 20 & $\uparrow$ & 22 & 22 & 22 & 21 & 21.8 & 26.69 \\
\hline 21 & $\rightarrow$ & 19 & 19 & 19 & 19 & 19.0 & 23.69 \\
\hline 22 & $\rightarrow$ & 18 & 18 & 18 & 18 & 18.0 & 23.67 \\
\hline 23 & $\rightarrow$ & 18 & 18 & 18 & 18 & 18.0 & 22.52 \\
\hline 24 & $\rightarrow$ & 18 & 18 & 18 & 18 & 18.0 & 22.80 \\
\hline 25 & $\rightarrow$ & 17 & 17 & 17 & 17 & 17.0 & 23.55 \\
\hline 26 & $\rightarrow$ & 17.5 & 17.5 & 17.5 & 17.5 & 17.5 & 25.31 \\
\hline 27 & $\rightarrow$ & 17 & 17.5 & 17.5 & 17 & 17.3 & 22.13 \\
\hline 28 & $\rightarrow$ & 17 & 17 & 17 & 16.5 & 16.9 & 23.10 \\
\hline 29 & $\rightarrow$ & 17 & 17 & 17 & 17.5 & 17.1 & 23.30 \\
\hline 30 & $\rightarrow$ & 18 & 18 & 18 & 18 & 18.0 & 24.71 \\
\hline
\end{tabular}


Table 7

Experimental data obtained for C25 concrete samples - Set 2

\begin{tabular}{|c|c|c|c|c|c|c|c|}
\hline Item & Position & 11 & 12 & 13 & 14 & la & $f_{c}(M P a)$ \\
\hline 31 & $\downarrow$ & 17 & 17 & 17 & 17 & 17.0 & 25.02 \\
\hline 32 & $\downarrow$ & 17 & 17 & 17 & 17 & 17.0 & 22.98 \\
\hline 33 & $\downarrow$ & 17 & 17 & 17 & 17 & 17.0 & 25.07 \\
\hline 34 & $\downarrow$ & 18 & 18 & 18 & 18 & 18.0 & 24.01 \\
\hline 35 & $\downarrow$ & 18 & 18 & 17 & 17 & 17.5 & 23.39 \\
\hline 36 & $\downarrow$ & 18 & 18 & 17.5 & 17 & 17.6 & 24.50 \\
\hline 37 & $\downarrow$ & 18 & 18 & 18 & $28^{* *}$ & 18.0 & 21.93 \\
\hline 38 & $\downarrow$ & 19 & 19 & 19 & 19 & 19.0 & 22.57 \\
\hline 39 & $\downarrow$ & 19 & 19 & 18 & 18 & 18.5 & 24.76 \\
\hline 40 & $\downarrow$ & 17 & 17 & 16 & 16 & 16.5 & 23.05 \\
\hline 41 & $\uparrow$ & 20 & 20 & 20 & 20 & 20.0 & 23.98 \\
\hline 42 & $\uparrow$ & 22 & 22 & 22 & 22 & 22.0 & 23.35 \\
\hline 43 & $\uparrow$ & 22 & 22 & 22 & 22 & 22.0 & 23.43 \\
\hline 44 & $\uparrow$ & 21 & 21 & 21 & 21 & 21.0 & 24.70 \\
\hline 45 & $\uparrow$ & 21 & 21 & 22 & 22 & 21.5 & 24.60 \\
\hline 46 & $\uparrow$ & 21 & 21 & 20.5 & 20 & 20.6 & 22.08 \\
\hline 47 & $\uparrow$ & 21 & 21 & 22 & 22 & 21.5 & 23.31 \\
\hline 48 & $\uparrow$ & 21 & 21 & 21 & 21 & 21.0 & 24.99 \\
\hline 49 & $\uparrow$ & 22 & 22 & 22 & 22 & 22.0 & 25.50 \\
\hline 50 & $\uparrow$ & 18 & 18 & 18 & 18 & 18.0 & 22.56 \\
\hline 51 & $\rightarrow$ & 19 & 19 & 19 & 19 & 19.0 & 24.70 \\
\hline 52 & $\rightarrow$ & 18 & 18 & 18 & 18 & 18.0 & 22.12 \\
\hline 53 & $\rightarrow$ & 19 & 19 & 19 & 19 & 19.0 & 24.00 \\
\hline 54 & $\rightarrow$ & 20 & 20 & 20 & 20 & 20.0 & 22.96 \\
\hline 55 & $\rightarrow$ & 18 & 18 & 18 & 18 & 18.0 & 22.74 \\
\hline 56 & $\rightarrow$ & 18 & 18 & 18 & 18 & 18.0 & 23.61 \\
\hline 57 & $\rightarrow$ & 18 & 18 & 19 & 19 & 18.5 & 23.54 \\
\hline 58 & $\rightarrow$ & 20 & 20 & 19.5 & 19.5 & 19.8 & 24.06 \\
\hline 59 & $\rightarrow$ & 20 & 20 & 21 & 21 & 20.5 & 23.39 \\
\hline 60 & $\rightarrow$ & 17 & 17 & 17 & 17 & 17.0 & 24.54 \\
\hline
\end{tabular}


Table 8

Experimental data obtained for C25 concrete samples - Set 3

\begin{tabular}{|c|c|c|c|c|c|c|c|}
\hline Item & Position & 11 & 12 & 13 & 14 & la & $f_{c}(M P a)$ \\
\hline 61 & $\downarrow$ & 18 & 18 & 18 & 18 & 18.0 & 23.69 \\
\hline 62 & $\downarrow$ & 18 & 18 & 18 & 18 & 18.0 & 22.75 \\
\hline 63 & $\downarrow$ & 18 & 18 & 18 & 18 & 18.0 & 23.95 \\
\hline 64 & $\downarrow$ & 17 & 17 & 17 & 17 & 17.0 & 24.37 \\
\hline 65 & $\downarrow$ & 17 & 17 & 18 & 18 & 17.5 & 22.91 \\
\hline 66 & $\downarrow$ & 18 & 18 & 17.5 & 17.5 & 17.8 & 25.02 \\
\hline 67 & $\downarrow$ & 19 & 19 & 20 & 20 & 19.5 & 24.87 \\
\hline 68 & $\downarrow$ & 19 & 19 & 19 & 19 & 19.0 & 25.71 \\
\hline 69 & $\downarrow$ & 19 & 19 & 19 & 19 & 19.0 & 25.24 \\
\hline 70 & $\downarrow$ & 19 & 19 & 19 & $15^{* *}$ & 19.0 & 22.05 \\
\hline 71 & $\uparrow$ & 21 & 21 & 21 & 21 & 21.0 & 25.78 \\
\hline 72 & $\uparrow$ & 21 & 21 & 21 & 21 & 21.0 & 23.24 \\
\hline 73 & $\uparrow$ & 21 & 21 & 21 & 21 & 21.0 & 25.30 \\
\hline 74 & $\uparrow$ & 20 & 20 & 20 & 20 & 20.0 & 22.12 \\
\hline 75 & $\uparrow$ & 20 & 20 & 21 & 21 & 20.5 & 25.27 \\
\hline 76 & $\uparrow$ & 22 & 22 & 23 & 23 & 22.5 & 23.86 \\
\hline 77 & $\uparrow$ & 20 & 20 & 21 & 21 & 20.5 & 24.92 \\
\hline 78 & $\uparrow$ & 22 & 22 & 22 & 22 & 22.0 & 23.20 \\
\hline 79 & $\uparrow$ & 21 & 21 & 21 & 21 & 21.0 & 25.35 \\
\hline 80 & $\uparrow$ & 18 & 18 & 18 & 18 & 18.0 & 25.34 \\
\hline 81 & $\rightarrow$ & 18 & 18 & 18 & 18 & 18.0 & 23.38 \\
\hline 82 & $\rightarrow$ & 18 & 18 & 18 & 18 & 18.0 & 24.13 \\
\hline 83 & $\rightarrow$ & 19 & 19 & 20 & 20 & 19.5 & 26.02 \\
\hline 84 & $\rightarrow$ & 20 & 20 & 19.5 & 19.5 & 19.8 & 25.46 \\
\hline 85 & $\rightarrow$ & 17 & 17 & 16.5 & 16.5 & 16.8 & 22.78 \\
\hline 86 & $\rightarrow$ & 16 & 16 & 16 & 16 & 16.0 & 26.67 \\
\hline 87 & $\rightarrow$ & 18 & 18 & 18 & 18 & 18.0 & 23.48 \\
\hline 88 & $\rightarrow$ & 16 & 18 & 16 & 18 & 17.0 & 22.49 \\
\hline 89 & $\rightarrow$ & 17 & 17 & 17 & 17 & 17.0 & 25.35 \\
\hline 90 & $\rightarrow$ & 19 & 19 & 19 & 19 & 19.0 & 24.06 \\
\hline
\end{tabular}


Table 9

Experimental data obtained for C30 concrete samples - Set 1

\begin{tabular}{|c|c|c|c|c|c|c|c|}
\hline Item & Position & 11 & 12 & 13 & 14 & la & $f_{c}(M P a)$ \\
\hline 1 & $\downarrow$ & 19 & 19 & 19 & 19 & 19.0 & 28.14 \\
\hline 2 & $\downarrow$ & 19 & 19 & 21 & 21 & 20.0 & 28.83 \\
\hline 3 & $\downarrow$ & 20 & 21 & 21 & 20 & 20.5 & 28.02 \\
\hline 4 & $\downarrow$ & 18 & 18 & 18 & 17.5 & 17.9 & 28.02 \\
\hline 5* & $\downarrow$ & 18 & 18 & 17.5 & 17.5 & 17.8 & 30.28 \\
\hline 6* & $\downarrow$ & 18 & 18 & 18 & 18 & 18.0 & 28.69 \\
\hline 7 & $\downarrow$ & 18 & 18 & 18 & 18 & 18.0 & 27.02 \\
\hline 8 & $\downarrow$ & 20 & 20 & 18 & 18 & 19.0 & 32.05 \\
\hline 9 & $\downarrow$ & 19 & 19 & 19 & 19 & 19.0 & 28.01 \\
\hline 10 & $\downarrow$ & 18.5 & 19 & 18.5 & 19 & 18.8 & 29.13 \\
\hline $11^{*}$ & $\uparrow$ & 22 & 22 & 23 & 23 & 22.5 & 28.62 \\
\hline 12 & $\uparrow$ & 24 & 24 & 23 & 23 & 23.5 & 28.93 \\
\hline 13 & $\uparrow$ & 23 & 23 & 23 & 23 & 23.0 & 27.95 \\
\hline 14 & $\uparrow$ & 23 & 23 & 23 & 23 & 23.0 & 32.19 \\
\hline 15 & $\uparrow$ & 24 & 24 & 22 & 22 & 23.0 & 32.65 \\
\hline 16 & $\uparrow$ & 24 & 24 & 24 & 24 & 24.0 & 26.88 \\
\hline 17 & $\uparrow$ & 24 & 24 & 24 & 24 & 24.0 & 33.82 \\
\hline 18 & $\uparrow$ & 22 & 22 & 21.5 & 21.5 & 21.8 & 29.63 \\
\hline 19 & $\uparrow$ & 21.5 & 22 & 21.5 & 22 & 21.8 & 34.33 \\
\hline 20 & $\uparrow$ & 22 & 22 & 22 & 22 & 22.0 & 30.84 \\
\hline 21 & $\rightarrow$ & 20 & 20 & 20 & 20 & 20.0 & 32.11 \\
\hline 22 & $\rightarrow$ & 20 & 20 & 20 & 20 & 20.0 & 29.20 \\
\hline 23 & $\rightarrow$ & 19 & 19 & 19 & 19 & 19.0 & 28.75 \\
\hline 24 & $\rightarrow$ & 19 & 19 & 19 & 19 & 19.0 & 29.35 \\
\hline 25 & $\rightarrow$ & 21 & 21 & 20 & 20 & 20.5 & 26.97 \\
\hline 26 & $\rightarrow$ & 21 & 21 & 20 & 20 & 20.5 & 27.02 \\
\hline 27 & $\rightarrow$ & 21 & 21 & 21 & 21 & 21.0 & 28.84 \\
\hline 28 & $\rightarrow$ & 20 & 20 & 19.5 & 19.5 & 19.8 & 31.37 \\
\hline 29 & $\rightarrow$ & 20 & 20 & 20 & 20 & 20.0 & 29.00 \\
\hline 30 & $\rightarrow$ & 19 & 19 & 19 & 19 & 19.0 & 34.43 \\
\hline
\end{tabular}




\section{Table 10}

Experimental data obtained for C20 concrete samples - Set 2

\begin{tabular}{|c|c|c|c|c|c|c|c|}
\hline Item & Position & 11 & 12 & 13 & 14 & la & $f_{c}(M P a)$ \\
\hline 31 & $\downarrow$ & 18 & 18 & 18 & 18 & 18.0 & 29.42 \\
\hline 32 & $\downarrow$ & 20 & 20 & 20 & 20 & 20.0 & 29.26 \\
\hline 33 & $\downarrow$ & 19 & 19 & 19 & 19 & 19.0 & 27.07 \\
\hline 34 & $\downarrow$ & 20 & 18 & 18 & 20 & 19.0 & 26.71 \\
\hline 35 & $\downarrow$ & 19 & 19 & 19 & 19 & 19.0 & 28.81 \\
\hline 36 & $\downarrow$ & 21 & 21 & 21 & 21 & 21.0 & 34.06 \\
\hline 37 & $\downarrow$ & 20 & 20 & 19.5 & 19.5 & 19.8 & 26.97 \\
\hline 38 & $\downarrow$ & 20 & 19.5 & 19.5 & 19.5 & 19.6 & 29.67 \\
\hline 39 & $\downarrow$ & 20 & 20 & 20 & 20 & 20.0 & 34.39 \\
\hline 40 & $\downarrow$ & 20 & 20 & 20 & 20 & 20.0 & 29.44 \\
\hline 41 & $\uparrow$ & 24 & 24 & 24 & 24 & 24.0 & 35.63 \\
\hline 42 & $\uparrow$ & 24 & 24 & 24 & 24 & 24.0 & 27.31 \\
\hline 43 & $\uparrow$ & 24 & 24 & 24 & 24 & 24.0 & 31.74 \\
\hline 44 & $\uparrow$ & 24 & 24 & 23 & 23 & 23.5 & 28.02 \\
\hline 45 & $\uparrow$ & 21 & 21 & 21 & 21 & 21.0 & 35.23 \\
\hline 46 & $\uparrow$ & 22 & 22 & 21 & 21 & 21.5 & 35.14 \\
\hline 47 & $\uparrow$ & 23 & 23 & 23 & 23 & 23.0 & 30.26 \\
\hline 48 & $\uparrow$ & 23 & 23 & 23 & 23 & 23.0 & 29.08 \\
\hline 49 & $\uparrow$ & 24 & 24 & 24 & 24 & 24.0 & 32.11 \\
\hline 50 & $\uparrow$ & 24 & 24 & 23 & 23 & 23.5 & 33.17 \\
\hline 51 & $\rightarrow$ & 20 & 20 & 20 & 20 & 20.0 & 31.22 \\
\hline 52 & $\rightarrow$ & 20 & 20 & 20 & 20 & 20.0 & 33.44 \\
\hline 53 & $\rightarrow$ & 19 & 19 & 21 & 21 & 20.0 & 32.19 \\
\hline 54 & $\rightarrow$ & 19 & 19 & 19 & 19 & 19.0 & 29.82 \\
\hline 55 & $\rightarrow$ & 19 & 19 & 19 & 19 & 19.0 & 29.69 \\
\hline 56 & $\rightarrow$ & 19 & 19 & 19 & 19 & 19.0 & 32.05 \\
\hline 57 & $\rightarrow$ & 23 & 23 & 22 & 22 & 22.5 & 32.12 \\
\hline 58 & $\rightarrow$ & 23 & 23 & 22.5 & 22.5 & 22.8 & 33.14 \\
\hline 59 & $\rightarrow$ & 21.5 & 21.5 & 21.5 & 22 & 21.6 & 29.09 \\
\hline 60 & $\rightarrow$ & 20 & 20 & 20 & 20 & 20.0 & 33.42 \\
\hline
\end{tabular}




\section{Table 11}

Experimental data obtained for 30 concrete samples - Set 3

\begin{tabular}{|c|c|c|c|c|c|c|c|}
\hline Item & Position & 11 & 12 & 13 & 14 & la & $f_{c}(M P a)$ \\
\hline 61 & $\downarrow$ & 19 & 19 & 19 & $14^{* *}$ & 19.0 & 29.36 \\
\hline 62 & $\downarrow$ & 19 & 19 & 19 & 19 & 19.0 & 26.88 \\
\hline 63 & $\downarrow$ & 19 & 19 & 19 & 19 & 19.0 & 32.91 \\
\hline 64 & $\downarrow$ & 20 & 20 & 20 & 20 & 20.0 & 28.14 \\
\hline 65 & $\downarrow$ & 20 & 20 & 20 & 20 & 20.0 & 29.34 \\
\hline 66 & $\downarrow$ & 20 & 20 & 20 & 20 & 20.0 & 31.23 \\
\hline 67 & $\downarrow$ & 18.5 & 18.5 & 19 & 19 & 18.8 & 32.38 \\
\hline 68 & $\downarrow$ & 18 & 18 & 18 & 18 & 18.0 & 31.36 \\
\hline 69 & $\downarrow$ & 18 & 18 & 18 & 18 & 18.0 & 31.61 \\
\hline 70 & $\downarrow$ & 19 & 19 & 19 & 19 & 19.0 & 32.63 \\
\hline 71 & $\uparrow$ & 23 & 23 & 23 & 23 & 23.0 & 31.89 \\
\hline 72 & $\uparrow$ & 23 & 23 & 23 & 23 & 23.0 & 30.71 \\
\hline 73 & $\uparrow$ & 23 & 23 & 23 & 23 & 23.0 & 32.66 \\
\hline 74 & $\uparrow$ & 24 & 24 & 24 & 24 & 24.0 & 31.59 \\
\hline 75 & $\uparrow$ & 25 & 25 & 25 & 25 & 25.0 & 27.12 \\
\hline 76 & $\uparrow$ & 24.5 & 24.5 & 24.5 & 24.5 & 24.5 & 28.79 \\
\hline 77 & $\uparrow$ & 24 & 24 & 24 & 24 & 24.0 & 27.22 \\
\hline 78 & $\uparrow$ & 23 & 22 & 22 & 22.5 & 22.4 & 27.51 \\
\hline 79 & $\uparrow$ & 23 & 22 & 22 & 22 & 22.3 & 32.07 \\
\hline 80 & $\uparrow$ & 22 & 22 & 22 & 22 & 22.0 & 29.53 \\
\hline 81 & $\rightarrow$ & 20 & 20 & 20 & 20 & 20.0 & 27.54 \\
\hline 82 & $\rightarrow$ & 20 & 20 & 20 & 20 & 20.0 & 31.87 \\
\hline 83 & $\rightarrow$ & 20 & 20 & 20 & 20 & 20.0 & 31.67 \\
\hline 84 & $\rightarrow$ & 19 & 19 & 19 & 19 & 19.0 & 28.34 \\
\hline 85 & $\rightarrow$ & 20 & 20 & 20 & 20 & 20.0 & 28.74 \\
\hline 86 & $\rightarrow$ & 19 & 19 & 19 & 19 & 19.0 & 33.19 \\
\hline 87 & $\rightarrow$ & 20 & 20 & 20 & 20 & 20.0 & 31.08 \\
\hline 88 & $\rightarrow$ & 20 & 20 & 20 & 20 & 20.0 & 32.06 \\
\hline 89 & $\rightarrow$ & 20 & 20 & 20 & 20 & 20.0 & 31.82 \\
\hline 90 & $\rightarrow$ & 19 & 19 & 19 & 19 & 19.0 & 31.95 \\
\hline
\end{tabular}


Table 12

Experimental data obtained for C35 concrete samples - Set 1

\begin{tabular}{|c|c|c|c|c|c|c|c|}
\hline Item & Position & 11 & 12 & 13 & 14 & la & $f_{c}(M P a)$ \\
\hline 1 & $\downarrow$ & 20 & 20 & 20 & 20 & 20.0 & 34.49 \\
\hline 2 & $\downarrow$ & 21 & 21 & 21 & 21 & 21.0 & 34.58 \\
\hline 3 & $\downarrow$ & 21 & 21 & 21 & 21 & 21.0 & 33.64 \\
\hline 4 & $\downarrow$ & 21 & 21 & 21 & 21 & 21.0 & 34.54 \\
\hline 5 & $\downarrow$ & 20 & 20 & 20 & 20 & 20.0 & 35.89 \\
\hline 6 & $\downarrow$ & 22 & 22 & 22 & 22 & 22.0 & 35.88 \\
\hline 7 & $\downarrow$ & 20 & 20 & 21 & 21 & 20.5 & 33.33 \\
\hline 8 & $\downarrow$ & 21 & 21 & 22 & 22 & 21.5 & 34.17 \\
\hline 9 & $\downarrow$ & 21.5 & 21.5 & 22 & 22 & 21.8 & 36.15 \\
\hline 10 & $\downarrow$ & 20 & 20 & 20 & 20 & 20.0 & 36.90 \\
\hline 11 & $\uparrow$ & 24 & 24 & 24 & 24 & 24.0 & 36.71 \\
\hline $12^{*}$ & $\uparrow$ & 24 & 24 & 24 & 24 & 24.0 & 34.10 \\
\hline 13 & $\uparrow$ & 25 & 25 & 25 & 25 & 25.0 & 35.04 \\
\hline 14 & $\uparrow$ & 25 & 25 & 25 & 25 & 25.0 & 34.29 \\
\hline 15 & $\uparrow$ & 27 & 27 & 27 & 27 & 27.0 & 34.67 \\
\hline 16 & $\uparrow$ & 27 & 27 & 27 & 27 & 27.0 & 32.34 \\
\hline 17 & $\uparrow$ & 25 & 25 & 25 & 25 & 25.0 & 34.10 \\
\hline 18 & $\uparrow$ & 24 & 24 & 24 & 24 & 24.0 & 32.24 \\
\hline 19 & $\uparrow$ & 24 & 24 & 24 & 24 & 24.0 & 36.11 \\
\hline 20 & $\uparrow$ & 24 & 24 & 24 & 24 & 24.0 & 35.59 \\
\hline 21 & $\rightarrow$ & 22 & 22 & 22 & 22 & 22.0 & 32.02 \\
\hline $22^{*}$ & $\rightarrow$ & 22 & 22 & 22 & 22 & 22.0 & 32.20 \\
\hline 23 & $\rightarrow$ & 22 & 22 & 22 & 22 & 22.0 & 35.63 \\
\hline $24^{*}$ & $\rightarrow$ & 21 & 21 & 21 & 21 & 21.0 & 37.70 \\
\hline 25 & $\rightarrow$ & 20 & 20 & 20 & 20 & 20.0 & 33.84 \\
\hline 26 & $\rightarrow$ & 23 & 22.5 & 22 & 22 & 22.4 & 32.09 \\
\hline 27 & $\rightarrow$ & 23 & 23 & 23 & 23 & 23.0 & 35.52 \\
\hline 28 & $\rightarrow$ & 23.5 & 23 & 23 & 23 & 23.1 & 34.98 \\
\hline 29 & $\rightarrow$ & 23 & 23 & 23 & 23 & 23.0 & 34.80 \\
\hline 30 & $\rightarrow$ & 22 & 22 & 22 & 22 & 22.0 & 37.93 \\
\hline
\end{tabular}


Table 13

Experimental data obtained for C35 concrete samples - Set 2

\begin{tabular}{|c|c|c|c|c|c|c|c|}
\hline Item & Position & 11 & 12 & 13 & 14 & la & $f_{c}(M P a)$ \\
\hline 31 & $\downarrow$ & 21 & 21 & 21 & 21 & 21.0 & 36.75 \\
\hline 32 & $\downarrow$ & 21 & 21 & 21 & $10^{* *}$ & 21.0 & 32.93 \\
\hline 33 & $\downarrow$ & 23 & 23 & 23 & 23 & 23.0 & 34.12 \\
\hline 34 & $\downarrow$ & 21 & 21 & 21 & 21 & 21.0 & 35.22 \\
\hline 35 & $\downarrow$ & 20 & 20 & 20 & 20 & 20.0 & 36.73 \\
\hline 36 & $\downarrow$ & 19 & 19 & 19 & 19 & 19.0 & 35.49 \\
\hline 37 & $\downarrow$ & 18 & 18 & 18 & 18 & 18.0 & 33.00 \\
\hline 38 & $\downarrow$ & 17 & 17 & 17 & 17 & 17.0 & 36.15 \\
\hline 39 & $\downarrow$ & 20 & 20 & 20 & 20 & 20.0 & 34.35 \\
\hline 40 & $\downarrow$ & 20 & 20 & 20 & 20 & 20.0 & 34.58 \\
\hline 41 & $\uparrow$ & 25 & 25 & 25 & 25 & 25.0 & 36.63 \\
\hline 42 & $\uparrow$ & 25 & 25 & 25 & 25 & 25.0 & 37.56 \\
\hline 43 & $\uparrow$ & 26 & 24 & 26 & 24 & 25.0 & 35.15 \\
\hline 44 & $\uparrow$ & 27 & 27 & 27 & 27 & 27.0 & 33.32 \\
\hline 45 & $\uparrow$ & 26 & 26 & 26 & 26 & 26.0 & 36.38 \\
\hline 46 & $\uparrow$ & 26 & 26 & 26 & 26 & 26.0 & 33.05 \\
\hline 47 & $\uparrow$ & 26 & 26 & 26 & 26 & 26.0 & 32.61 \\
\hline 48 & $\uparrow$ & 24 & 24 & 24 & 24 & 24.0 & 33.54 \\
\hline 49 & $\uparrow$ & 24 & 24 & 23.5 & 23.5 & 23.8 & 32.72 \\
\hline 50 & $\uparrow$ & 23 & 23 & 23 & 23 & 23.0 & 36.27 \\
\hline 51 & $\rightarrow$ & 23 & 23 & 23 & 23 & 23.0 & 32.84 \\
\hline 52 & $\rightarrow$ & 23 & 23 & 23 & 23 & 23.0 & 34.89 \\
\hline 53 & $\rightarrow$ & 23 & 23 & 23 & 23 & 23.0 & 36.63 \\
\hline 54 & $\rightarrow$ & 22 & 22 & 22 & 22 & 22.0 & 35.75 \\
\hline 55 & $\rightarrow$ & 21 & 21 & 21 & 21 & 21.0 & 32.84 \\
\hline 56 & $\rightarrow$ & 20 & 20 & 20 & 20 & 20.0 & 32.34 \\
\hline 57 & $\rightarrow$ & 20.5 & 20.5 & 21 & 21 & 20.8 & 32.37 \\
\hline 58 & $\rightarrow$ & 20 & 20 & 21 & 21 & 20.5 & 32.43 \\
\hline 59 & $\rightarrow$ & 22 & 22 & 22 & 22 & 22.0 & 36.86 \\
\hline 60 & $\rightarrow$ & 22 & 22 & 22 & 22 & 22.0 & 34.40 \\
\hline
\end{tabular}


Table 14

Experimental data obtained for 35 concrete samples - Set 3

\begin{tabular}{|c|c|c|c|c|c|c|c|}
\hline Item & Position & 11 & 12 & 13 & 14 & Ia & $f_{c}(\mathrm{MPa})$ \\
\hline 61 & $\downarrow$ & 22 & 22 & 22 & 22 & 22.0 & 34.11 \\
\hline 62 & $\downarrow$ & 19 & 19 & 19 & 19 & 19.0 & 36.90 \\
\hline 63 & $\downarrow$ & 21 & 21 & 19 & 19 & 20.0 & 36.90 \\
\hline 64 & $\downarrow$ & 20 & 20 & 20 & 20 & 20.0 & 35.80 \\
\hline 65 & $\downarrow$ & 20 & 20 & 20 & 20 & 20.0 & 34.45 \\
\hline 66 & $\downarrow$ & 22 & 22 & 22 & 22 & 22.0 & 36.27 \\
\hline 67 & $\downarrow$ & 22 & 22 & 20 & 20 & 21.0 & 32.20 \\
\hline 68 & $\downarrow$ & 20 & 20 & 20 & 20 & 20.0 & 34.82 \\
\hline 69 & $\downarrow$ & 20 & 20 & 20 & 20 & 20.0 & 34.39 \\
\hline 70 & $\downarrow$ & 20 & 20 & 20 & 20 & 20.0 & 33.61 \\
\hline 71 & $\uparrow$ & 25 & 25 & 25 & 25 & 25.0 & 36.39 \\
\hline 72 & $\uparrow$ & 25 & 25 & 25 & 25 & 25.0 & 33.51 \\
\hline 73 & $\uparrow$ & 25 & 25 & 25 & 25 & 25.0 & 35.27 \\
\hline 74 & $\uparrow$ & 24 & 24 & 24 & 24 & 24.0 & 34.29 \\
\hline 75 & $\uparrow$ & 24 & 24 & 25 & 25 & 24.5 & 34.28 \\
\hline 76 & $\uparrow$ & 26 & 26 & 26 & 26 & 26.0 & 32.31 \\
\hline 77 & $\uparrow$ & 26 & 26 & 26 & 26 & 26.0 & 34.43 \\
\hline 78 & $\uparrow$ & 24.5 & 24.5 & 25 & 25 & 24.8 & 35.97 \\
\hline 79 & $\uparrow$ & 25 & 25 & 25 & 25 & 25.0 & 36.58 \\
\hline 80 & $\uparrow$ & 25 & 25 & 25 & 25 & 25.0 & 34.78 \\
\hline 81 & $\rightarrow$ & 23 & 23 & 23 & 23 & 23.0 & 32.25 \\
\hline 82 & $\rightarrow$ & 21 & 23 & 23 & 21 & 22.0 & 35.99 \\
\hline 83 & $\rightarrow$ & 22 & 22 & 22 & 22 & 22.0 & 35.60 \\
\hline 84 & $\rightarrow$ & 22 & 22 & 22 & 22 & 22.0 & 33.38 \\
\hline 85 & $\rightarrow$ & 23 & 23 & 23 & 23 & 23.0 & 32.75 \\
\hline 86 & $\rightarrow$ & 23 & 23 & 23 & 23 & 23.0 & 36.72 \\
\hline 87 & $\rightarrow$ & 20 & 20 & 20 & 20 & 20.0 & 37.23 \\
\hline 88 & $\rightarrow$ & 21.5 & 21.5 & 21.5 & 21.5 & 21.5 & 34.64 \\
\hline 89 & $\rightarrow$ & 22 & 22 & 22 & 22 & 22.0 & 34.39 \\
\hline 90 & $\rightarrow$ & 20 & 20 & 21 & 21 & 20.5 & 36.05 \\
\hline
\end{tabular}


Table 15

Experimental data obtained for C40 concrete samples - Set 1

\begin{tabular}{|c|c|c|c|c|c|c|c|}
\hline Item & Position & 11 & 12 & 13 & 14 & la & $f_{c}(M P a)$ \\
\hline $1^{*}$ & $\downarrow$ & 23 & 23 & 23 & 23 & 23.0 & 37.96 \\
\hline 2 & $\downarrow$ & 23 & 23 & 24 & 24 & 23.5 & 40.65 \\
\hline 3 & $\downarrow$ & 23 & 23 & 23 & 23 & 23.0 & 36.38 \\
\hline 4 & $\downarrow$ & 23 & 23 & 23 & 23 & 23.0 & 38.25 \\
\hline 5 & $\downarrow$ & 24 & 24 & 24 & 24 & 24.0 & 41.14 \\
\hline 6 & $\downarrow$ & 25 & 25 & 25 & 25 & 25.0 & 37.64 \\
\hline 7 & $\downarrow$ & 25 & 25 & 25 & 25 & 25.0 & 39.72 \\
\hline 8 & $\downarrow$ & 25 & 25 & 25 & 25 & 25.0 & 41.42 \\
\hline 9* & $\downarrow$ & 24 & 24 & 25 & 25 & 24.5 & 27.49 \\
\hline 10 & $\downarrow$ & 23 & 23 & 23 & 23 & 23.0 & 42.41 \\
\hline 11 & $\uparrow$ & 27 & 27 & 27 & 27 & 27.0 & 39.43 \\
\hline 12 & $\uparrow$ & 27 & 27 & 29 & 29 & 28.0 & 39.89 \\
\hline 13 & $\uparrow$ & 30 & 30 & 30 & 30 & 30.0 & 39.80 \\
\hline 14 & $\uparrow$ & 30 & 30 & 30 & 30 & 30.0 & 38.38 \\
\hline 15 & $\uparrow$ & 30 & 30 & 30 & 30 & 30.0 & 38.80 \\
\hline 16 & $\uparrow$ & 29 & 29 & 29 & 29 & 29.0 & 39.94 \\
\hline 17 & $\uparrow$ & 29 & 29 & 29 & 29 & 29.0 & 39.04 \\
\hline 18 & $\uparrow$ & 27 & 27 & 28 & 28 & 27.5 & 39.11 \\
\hline 19 & $\uparrow$ & 29 & 29 & 28.5 & 28 & 28.6 & 42.14 \\
\hline 20 & $\uparrow$ & 29 & 29 & 29 & 29 & 29.0 & 40.48 \\
\hline 21 & $\rightarrow$ & 26 & 26 & 26 & 26 & 26.0 & 39.16 \\
\hline 22 & $\rightarrow$ & 26 & 26 & 26 & 26 & 26.0 & 39.60 \\
\hline $23^{*}$ & $\rightarrow$ & 28 & 28 & 27 & 27 & 27.5 & 39.70 \\
\hline 24 & $\rightarrow$ & 28.5 & 28.5 & 27 & 27 & 27.8 & 40.25 \\
\hline 25 & $\rightarrow$ & 28 & 28 & 28 & 28 & 28.0 & 40.77 \\
\hline 26 & $\rightarrow$ & 27 & 27 & 29 & 29 & 28.0 & 39.14 \\
\hline $27^{*}$ & $\rightarrow$ & 28 & 28 & 28 & 28 & 28.0 & 38.58 \\
\hline 28 & $\rightarrow$ & 27 & 27 & 27 & 27 & 27.0 & 38.64 \\
\hline 29 & $\rightarrow$ & 27 & 27 & 27 & 27 & 27.0 & 39.56 \\
\hline 30 & $\rightarrow$ & 28 & 28 & 28 & 28 & 28.0 & 42.62 \\
\hline
\end{tabular}


Table 16

Experimental data obtained for C40 concrete samples - Set 2

\begin{tabular}{|c|c|c|c|c|c|c|c|}
\hline Item & Position & 11 & 12 & 13 & 14 & la & $f_{c}(M P a)$ \\
\hline 31 & $\downarrow$ & 23 & 23 & 23 & 23 & 23.0 & 38.06 \\
\hline 32 & $\downarrow$ & 23 & 23 & 23 & 23 & 23.0 & 41.85 \\
\hline 33 & $\downarrow$ & 23 & 23 & 23 & 23 & 23.0 & 38.55 \\
\hline 34 & $\downarrow$ & 24 & 24 & 24 & 24 & 24.0 & 38.86 \\
\hline 35 & $\downarrow$ & 24 & 24 & 24 & 24 & 24.0 & 39.13 \\
\hline 36 & $\downarrow$ & 24 & 24 & 24 & 24 & 24.0 & 38.94 \\
\hline 37 & $\downarrow$ & 25 & 25 & 25 & 25 & 25.0 & 38.82 \\
\hline 38 & $\downarrow$ & 25.5 & 25.5 & 25.5 & 25.5 & 25.5 & 39.05 \\
\hline 39 & $\downarrow$ & 26 & 26 & 24 & 24 & 25.0 & 39.44 \\
\hline 40 & $\downarrow$ & 24 & 24 & 24 & 24 & 24.0 & 42.08 \\
\hline 41 & $\uparrow$ & 29 & 29 & 29 & 29 & 29.0 & 43.06 \\
\hline 42 & $\uparrow$ & 29 & 29 & 29 & 29 & 29.0 & 39.38 \\
\hline 43 & $\uparrow$ & 29 & 29 & 29 & 29 & 29.0 & 41.80 \\
\hline 44 & $\uparrow$ & 28 & 28 & 28 & 28 & 28.0 & 38.55 \\
\hline 45 & $\uparrow$ & 28 & 28 & 28 & 28 & 28.0 & 38.55 \\
\hline 46 & $\uparrow$ & 28 & 28 & 28 & 28 & 28.0 & 38.91 \\
\hline 47 & $\uparrow$ & 28 & 28 & 27 & 27 & 27.5 & 38.48 \\
\hline 48 & $\uparrow$ & 27 & 28 & 28 & 27 & 27.5 & 39.55 \\
\hline 49 & $\uparrow$ & 27 & 27 & 27 & 27 & 27.0 & 40.41 \\
\hline 50 & $\uparrow$ & 27 & 27 & 27 & 27 & 27.0 & 38.46 \\
\hline 51 & $\rightarrow$ & 26 & 26 & 26 & 26 & 26.0 & 41.47 \\
\hline 52 & $\rightarrow$ & 26 & 26 & 26 & 26 & 26.0 & 42.59 \\
\hline 53 & $\rightarrow$ & 26 & 26 & 26 & 26 & 26.0 & 38.90 \\
\hline 54 & $\rightarrow$ & 26 & 26 & 26 & 26 & 26.0 & 38.63 \\
\hline 55 & $\rightarrow$ & 26 & 26 & 26 & 26 & 26.0 & 39.20 \\
\hline 56 & $\rightarrow$ & 28 & 28 & 27 & 27 & 27.5 & 39.13 \\
\hline 57 & $\rightarrow$ & 28 & 27.5 & 27.5 & 27.5 & 27.6 & 40.91 \\
\hline 58 & $\rightarrow$ & 28 & 28 & 28 & 28 & 28.0 & 38.73 \\
\hline 59 & $\rightarrow$ & 28 & 28 & 28 & 28 & 28.0 & 40.41 \\
\hline 60 & $\rightarrow$ & 28 & 28 & 28 & 28 & 28.0 & 40.54 \\
\hline
\end{tabular}




\section{Table 17}

Experimental data obtained for 40 concrete samples - Set 3

\begin{tabular}{|c|c|c|c|c|c|c|c|}
\hline Item & Position & 11 & 12 & 13 & 14 & la & $f_{c}(M P a)$ \\
\hline 61 & $\downarrow$ & 24 & 24 & 24 & 24 & 24.0 & 39.13 \\
\hline 62 & $\downarrow$ & 24 & 24 & 24 & 24 & 24.0 & 38.62 \\
\hline 63 & $\downarrow$ & 24 & 24 & 24 & 24 & 24.0 & 39.43 \\
\hline 64 & $\downarrow$ & 24 & 24 & 24 & 24 & 24.0 & 40.21 \\
\hline 65 & $\downarrow$ & 24 & 24 & 23 & 23 & 23.5 & 42.91 \\
\hline 66 & $\downarrow$ & 24 & 23.5 & 23.5 & 23.5 & 23.6 & 38.29 \\
\hline 67 & $\downarrow$ & 23.5 & 23.5 & 23.5 & 24 & 23.6 & 39.20 \\
\hline 68 & $\downarrow$ & 25 & 25 & 25 & 25 & 25.0 & 38.46 \\
\hline 69 & $\downarrow$ & 25 & 25 & 25 & 25 & 25.0 & 38.81 \\
\hline 70 & $\downarrow$ & 24 & 24 & 24 & 24 & 24.0 & 41.67 \\
\hline 71 & $\uparrow$ & 29 & 29 & 29 & 29 & 29.0 & 38.82 \\
\hline 72 & $\uparrow$ & 29 & 29 & 29 & 29 & 29.0 & 38.90 \\
\hline 73 & $\uparrow$ & 29 & 29 & 29 & 29 & 29.0 & 38.76 \\
\hline 74 & $\uparrow$ & 29 & 29 & 29 & 29 & 29.0 & 38.58 \\
\hline 75 & $\uparrow$ & 31 & 31 & 31 & 31 & 31.0 & 40.20 \\
\hline 76 & $\uparrow$ & 31 & 31 & 29 & 29 & 30.0 & 41.65 \\
\hline 77 & $\uparrow$ & 27 & 27 & 27 & 27 & 27.0 & 38.78 \\
\hline 78 & $\uparrow$ & 26 & 26 & 26 & 26 & 26.0 & 38.31 \\
\hline 79 & $\uparrow$ & 26 & 26 & 26 & 26 & 26.0 & 43.21 \\
\hline 80 & $\uparrow$ & 26 & 26 & 26 & 26 & 26.0 & 39.99 \\
\hline 81 & $\rightarrow$ & 28 & 28 & 26 & 26 & 27.0 & 39.20 \\
\hline 82 & $\rightarrow$ & 27 & 27 & 27 & 27 & 27.0 & 42.74 \\
\hline 83 & $\rightarrow$ & 27 & 27 & 27 & 27 & 27.0 & 40.59 \\
\hline 84 & $\rightarrow$ & 29 & 29 & 29 & 29 & 29.0 & 43.11 \\
\hline 85 & $\rightarrow$ & 28 & 28 & 28 & 28 & 28.0 & 42.07 \\
\hline 86 & $\rightarrow$ & 28 & 28 & 28 & 28 & 28.0 & 39.22 \\
\hline 87 & $\rightarrow$ & 28 & 28 & 28 & 28 & 28.0 & 38.39 \\
\hline 88 & $\rightarrow$ & 29 & 29 & 29 & 29 & 29.0 & 40.29 \\
\hline 89 & $\rightarrow$ & 29 & 29 & 29 & 29 & 29.0 & 41.13 \\
\hline 90 & $\rightarrow$ & 26 & 26 & 26 & 26 & 26.0 & 42.70 \\
\hline
\end{tabular}


Table 18

Experimental data obtained for C45 concrete samples - Set 1

\begin{tabular}{|c|c|c|c|c|c|c|c|}
\hline Item & Position & 11 & 12 & 13 & 14 & la & $f_{c}(M P a)$ \\
\hline $1 *$ & $\downarrow$ & 28 & 28 & 28 & 28 & 28.0 & 44.69 \\
\hline 2 & $\downarrow$ & 28 & 28 & 28 & 28 & 28.0 & 44.67 \\
\hline 3 & $\downarrow$ & 29 & 29 & 29 & 29 & 29.0 & 45.16 \\
\hline 4 & $\downarrow$ & 29 & 29 & 29 & 29 & 29.0 & 45.10 \\
\hline 5 & $\downarrow$ & 29 & 29 & 29 & 29 & 29.0 & 45.31 \\
\hline 6 & $\downarrow$ & 31 & 31 & 31 & 31 & 31.0 & 46.42 \\
\hline 7 & $\downarrow$ & 31 & 31 & 31 & 31 & 31.0 & 46.84 \\
\hline 8 & $\downarrow$ & 31 & 31 & 31 & 31 & 31.0 & 44.81 \\
\hline 9 & $\downarrow$ & 31 & 31 & 29 & 29 & 30.0 & 44.56 \\
\hline 10 & $\downarrow$ & 30 & 30 & 30 & 30 & 30.0 & 45.59 \\
\hline 11 & $\uparrow$ & 32 & 32 & 32 & 32 & 32.0 & 48.32 \\
\hline 12 & $\uparrow$ & 32 & 31.5 & 32 & $15^{* *}$ & 31.8 & 43.57 \\
\hline 13 & $\uparrow$ & 31.5 & 31.5 & 32 & 31.5 & 31.6 & 45.45 \\
\hline $14^{*}$ & $\uparrow$ & 32 & 32 & 32 & 32 & 32.0 & 47.58 \\
\hline 15 & $\uparrow$ & 33 & 33 & 33 & 33 & 33.0 & 46.54 \\
\hline 16 & $\uparrow$ & 33 & 33 & 33 & 33 & 33.0 & 46.19 \\
\hline 17 & $\uparrow$ & 33 & 33 & 33 & 33 & 33.0 & 42.79 \\
\hline 18 & $\uparrow$ & 34 & 34 & 34 & 34 & 34.0 & 43.23 \\
\hline 19 & $\uparrow$ & 34 & 34 & 33.5 & 33.5 & 33.8 & 42.53 \\
\hline 20 & $\uparrow$ & 34 & 34 & 34 & 34 & 34.0 & 44.58 \\
\hline 21 & $\rightarrow$ & 31 & 31 & 31 & 31 & 31.0 & 45.06 \\
\hline 22 & $\rightarrow$ & 31 & 31 & 31 & 31 & 31.0 & 47.99 \\
\hline $23^{*}$ & $\rightarrow$ & 31 & 31 & 31 & 31 & 31.0 & 48.00 \\
\hline 24 & $\rightarrow$ & 32 & 32 & 32 & 32 & 32.0 & 45.58 \\
\hline 25 & $\rightarrow$ & 32 & 32 & 32 & 32 & 32.0 & 48.79 \\
\hline 26 & $\rightarrow$ & 32 & 32 & 32 & 32 & 32.0 & 43.00 \\
\hline $27^{*}$ & $\rightarrow$ & 33 & 33 & 33 & 33 & 33.0 & 45.42 \\
\hline 28 & $\rightarrow$ & 33.5 & 33.5 & 32 & 32.5 & 32.9 & 44.64 \\
\hline 29 & $\rightarrow$ & 33 & 33 & 32.5 & 32.5 & 32.8 & 45.54 \\
\hline 30 & $\rightarrow$ & 32 & 32 & 32 & 32 & 32.0 & 43.99 \\
\hline
\end{tabular}


Table 19

Experimental data obtained for C45 concrete samples - Set 2

\begin{tabular}{|c|c|c|c|c|c|c|c|}
\hline Item & Position & 11 & 12 & 13 & 14 & la & $f_{c}(M P a)$ \\
\hline 31 & $\downarrow$ & 30 & 30 & 30 & 30 & 30.0 & 47.44 \\
\hline 32 & $\downarrow$ & 30 & 30 & 30 & 30 & 30.0 & 46.43 \\
\hline 33 & $\downarrow$ & 30 & 30 & 30 & 30 & 30.0 & 44.63 \\
\hline 34 & $\downarrow$ & 30 & 30 & 30 & 30 & 30.0 & 44.58 \\
\hline 35 & $\downarrow$ & 30 & 30 & 30 & 30 & 30.0 & 45.82 \\
\hline 36 & $\downarrow$ & 30 & 30 & 28 & 28 & 29.0 & 46.57 \\
\hline 37 & $\downarrow$ & 29 & 29 & 29 & 29 & 29.0 & 45.61 \\
\hline 38 & $\downarrow$ & 29 & 29 & 29 & 29 & 29.0 & 44.95 \\
\hline 39 & $\downarrow$ & 31 & 31 & 31 & 31 & 31.0 & 47.76 \\
\hline 40 & $\downarrow$ & 31.5 & 32 & 32 & 31.5 & 31.8 & 46.72 \\
\hline 41 & $\uparrow$ & 32 & 32 & 32 & 32 & 32.0 & 47.86 \\
\hline 42 & $\uparrow$ & 33 & 33 & 33 & 33 & 33.0 & 44.79 \\
\hline 43 & $\uparrow$ & 33 & 33 & 33 & 33 & 33.0 & 46.69 \\
\hline 44 & $\uparrow$ & 33 & 33 & 33 & 33 & 33.0 & 45.16 \\
\hline 45 & $\uparrow$ & 34 & 34 & 34 & 34 & 34.0 & 44.42 \\
\hline 46 & $\uparrow$ & 32 & 32 & 32 & 32 & 32.0 & 43.49 \\
\hline 47 & $\uparrow$ & 32 & 32 & 32 & 32 & 32.0 & 48.04 \\
\hline 48 & $\uparrow$ & 34 & 34 & 34 & 34 & 34.0 & 45.51 \\
\hline 49 & $\uparrow$ & 34 & 34 & 34 & 34 & 34.0 & 46.92 \\
\hline 50 & $\uparrow$ & 34 & 32 & 34 & 32 & 33.0 & 47.84 \\
\hline 51 & $\rightarrow$ & 32 & 32 & 32 & 32 & 32.0 & 45.39 \\
\hline 52 & $\rightarrow$ & 32 & 32 & 32 & 32 & 32.0 & 48.46 \\
\hline 53 & $\rightarrow$ & 32 & 32 & 32 & 32 & 32.0 & 45.80 \\
\hline 54 & $\rightarrow$ & 32 & 32 & 32 & 32 & 32.0 & 45.02 \\
\hline 55 & $\rightarrow$ & 32 & 32 & 32 & 32 & 32.0 & 46.75 \\
\hline 56 & $\rightarrow$ & 33 & 33 & 32.5 & 32.5 & 32.8 & 45.39 \\
\hline 57 & $\rightarrow$ & 33 & 33 & 33 & 32.5 & 32.9 & 45.52 \\
\hline 58 & $\rightarrow$ & 31 & 31 & 32 & 32 & 31.5 & 48.24 \\
\hline 59 & $\rightarrow$ & 31 & 31 & 31.5 & 31.5 & 31.3 & 46.23 \\
\hline 60 & $\rightarrow$ & 31 & 31 & 31 & 31 & 31.0 & 47.48 \\
\hline
\end{tabular}


Table 20

Experimental data obtained for C45 concrete samples - Set 3

\begin{tabular}{|c|c|c|c|c|c|c|c|}
\hline Item & Position & 11 & 12 & 13 & 14 & Ia & $f_{c}(\mathrm{MPa})$ \\
\hline 61 & $\downarrow$ & 30 & 30 & 30 & 30 & 30.0 & 45.16 \\
\hline 62 & $\downarrow$ & 30 & 30 & 30 & 30 & 30.0 & 45.25 \\
\hline 63 & $\downarrow$ & 30 & 30 & 30 & 30 & 30.0 & 44.59 \\
\hline 64 & $\downarrow$ & 29 & 29 & 29 & 29 & 29.0 & 45.79 \\
\hline 65 & $\downarrow$ & 29 & 29 & 29 & 29 & 29.0 & 46.37 \\
\hline 66 & $\downarrow$ & 29 & 29 & 29 & 29 & 29.0 & 48.17 \\
\hline 67 & $\downarrow$ & 29 & 29 & 29 & 29 & 29.0 & 47.53 \\
\hline 68 & $\downarrow$ & 31 & 31 & 30.5 & 30.5 & 30.8 & 45.45 \\
\hline 69 & $\downarrow$ & 31 & 30.5 & 30.5 & 30.5 & 30.6 & 48.27 \\
\hline 70 & $\downarrow$ & 31 & 31 & 31 & 31 & 31.0 & 45.99 \\
\hline 71 & $\uparrow$ & 33 & 33 & 33 & 33 & 33.0 & 47.20 \\
\hline 72 & $\uparrow$ & 33 & 33 & 33 & 33 & 33.0 & 45.23 \\
\hline 73 & $\uparrow$ & 33 & 33 & 33 & 33 & 33.0 & 46.96 \\
\hline 74 & $\uparrow$ & 33 & 33 & 33 & 33 & 33.0 & 45.28 \\
\hline 75 & $\uparrow$ & 33 & 33 & 33 & 33 & 33.0 & 45.58 \\
\hline 76 & $\uparrow$ & 33 & 33 & 31 & 31 & 32.0 & 43.93 \\
\hline 77 & $\uparrow$ & 32 & 32 & 32 & 32 & 32.0 & 42.36 \\
\hline 78 & $\uparrow$ & 33 & 33 & 33 & 33 & 33.0 & 44.73 \\
\hline 79 & $\uparrow$ & 33 & 33 & 33 & 33 & 33.0 & 46.40 \\
\hline 80 & $\uparrow$ & 32 & 32 & 32 & 32 & 32.0 & 42.88 \\
\hline 81 & $\rightarrow$ & 32 & 32 & 32 & 32 & 32.0 & 45.07 \\
\hline 82 & $\rightarrow$ & 32 & 32 & 32 & 32 & 32.0 & 46.68 \\
\hline 83 & $\rightarrow$ & 30 & 30 & 30 & 30 & 30.0 & 45.86 \\
\hline 84 & $\rightarrow$ & 30 & 30 & 30 & 30 & 30.0 & 45.28 \\
\hline 85 & $\rightarrow$ & 30 & 30 & 30 & 30 & 30.0 & 45.62 \\
\hline 86 & $\rightarrow$ & 30 & 30 & 30 & 30 & 30.0 & 47.25 \\
\hline 87 & $\rightarrow$ & 32 & 32 & 33 & 33 & 32.5 & 47.16 \\
\hline 88 & $\rightarrow$ & 33 & 33 & 33 & 33 & 33.0 & 47.66 \\
\hline 89 & $\rightarrow$ & 33 & 33 & 33 & 33 & 33.0 & 45.70 \\
\hline 90 & $\rightarrow$ & 33 & 33 & 33 & 33 & 33.0 & 45.20 \\
\hline
\end{tabular}




\section{Table 21}

Experimental data obtained for C50 concrete samples - Set 1

\begin{tabular}{|c|c|c|c|c|c|c|c|}
\hline Item & Position & 11 & 12 & 13 & 14 & la & $f_{c}(M P a)$ \\
\hline 1 & $\downarrow$ & 32 & 32 & 32 & 32 & 32.0 & 50.87 \\
\hline 2 & $\downarrow$ & 32 & 32 & 32 & 32 & 32.0 & 47.30 \\
\hline 3 & $\downarrow$ & 32 & 32 & 32 & 32 & 32.0 & 48.55 \\
\hline 4 & $\downarrow$ & 32 & 32 & 32 & 32 & 32.0 & 49.19 \\
\hline 5 & $\downarrow$ & 32 & 32 & 30 & 30 & 31.0 & 49.21 \\
\hline 6 & $\downarrow$ & 30 & 30 & 30 & 30 & 30.0 & 56.84 \\
\hline 7* & $\downarrow$ & 33 & 33 & 33 & 33 & 33.0 & 54.77 \\
\hline 8 & $\downarrow$ & 33 & 33 & 33 & 33 & 33.0 & 47.57 \\
\hline 9 & $\downarrow$ & 32 & 32 & 32 & 32 & 32.0 & 55.41 \\
\hline 10 & $\downarrow$ & 33 & 33 & 31 & 31 & 32.0 & 53.34 \\
\hline 11 & $\uparrow$ & 35 & 35 & 35 & 35 & 35.0 & 47.57 \\
\hline 12 & $\uparrow$ & 33 & 33 & 33 & 33 & 33.0 & 54.79 \\
\hline $13^{*}$ & $\uparrow$ & 35 & 35 & 35 & 35 & 35.0 & 54.81 \\
\hline 14 & $\uparrow$ & 35 & 35 & 33 & 33 & 34.0 & 48.73 \\
\hline 15 & $\uparrow$ & 36 & 36 & 36 & 36 & 36.0 & 48.13 \\
\hline 16 & $\uparrow$ & 36 & 36 & 36 & 36 & 36.0 & 48.78 \\
\hline $17^{*}$ & $\uparrow$ & 35 & 35 & 35 & 35 & 35.0 & 33.50 \\
\hline 18 & $\uparrow$ & 34 & 34 & 34 & 34 & 34.0 & 50.57 \\
\hline 19 & $\uparrow$ & 36 & 36 & 34 & 34 & 35.0 & 48.47 \\
\hline 20 & $\uparrow$ & 35 & 35 & 35 & 35 & 35.0 & 49.31 \\
\hline 21 & $\rightarrow$ & 33 & 33 & 33 & 33 & 33.0 & 47.47 \\
\hline 22 & $\rightarrow$ & 33 & 33 & 33 & 33 & 33.0 & 49.25 \\
\hline $23^{*}$ & $\rightarrow$ & 34 & 34 & 32 & 32 & 33.0 & 53.86 \\
\hline 24 & $\rightarrow$ & 34 & 34 & 34 & 34 & 34.0 & 53.65 \\
\hline 25 & $\rightarrow$ & 32 & 32 & 32 & 32 & 32.0 & 50.03 \\
\hline 26 & $\rightarrow$ & 34 & 34 & 34 & 34 & 34.0 & 49.20 \\
\hline $27^{*}$ & $\rightarrow$ & 24 & 24 & 22 & 22 & 23.0 & 31.28 \\
\hline 28 & $\rightarrow$ & 31 & 31 & 31 & 31 & 31.0 & 43.02 \\
\hline 29 & $\rightarrow$ & 33 & 33 & 31 & 31 & 32.0 & 41.97 \\
\hline 30 & $\rightarrow$ & 31 & 31 & 31 & 31 & 31.0 & 47.36 \\
\hline
\end{tabular}


Table 22

Experimental data obtained for C50 concrete samples - Set 2

\begin{tabular}{|c|c|c|c|c|c|c|c|}
\hline Item & Position & 11 & 12 & 13 & 14 & la & $f_{c}(M P a)$ \\
\hline 31 & $\downarrow$ & 33 & 33 & 33 & 33 & 33.0 & 55.88 \\
\hline 32 & $\downarrow$ & 33 & 33 & 33 & 33 & 33.0 & 51.66 \\
\hline 33 & $\downarrow$ & 33 & 33 & 31 & 31 & 32.0 & 48.87 \\
\hline 34 & $\downarrow$ & 31 & 31 & 31 & 31 & 31.0 & 54.57 \\
\hline 35 & $\downarrow$ & 32 & 32 & 32 & 32 & 32.0 & 51.26 \\
\hline 36 & $\downarrow$ & 32 & 32 & 32 & 32 & 32.0 & 56.26 \\
\hline 37 & $\downarrow$ & 32 & 32 & 32 & 32 & 32.0 & 48.52 \\
\hline 38 & $\downarrow$ & 31 & 31 & 31 & 31 & 31.0 & 56.93 \\
\hline 39 & $\downarrow$ & 33 & 33 & 33 & 33 & 33.0 & 52.01 \\
\hline 40 & $\downarrow$ & 33 & 31 & 33 & 31 & 32.0 & 55.72 \\
\hline 41 & $\uparrow$ & 34 & 34 & 34 & 34 & 34.0 & 50.88 \\
\hline 42 & $\uparrow$ & 34 & 34 & 34 & 34 & 34.0 & 48.92 \\
\hline 43 & $\uparrow$ & 34 & 34 & 34 & 34 & 34.0 & 48.19 \\
\hline 44 & $\uparrow$ & 34 & 34 & 34 & 34 & 34.0 & 52.48 \\
\hline 45 & $\uparrow$ & 34 & 34 & 36 & 36 & 35.0 & 48.70 \\
\hline 46 & $\uparrow$ & 36 & 36 & 36 & 36 & 36.0 & 47.43 \\
\hline 47 & $\uparrow$ & 36 & 36 & 36 & 36 & 36.0 & 49.90 \\
\hline 48 & $\uparrow$ & 35 & 35 & 35 & 35 & 35.0 & 48.43 \\
\hline 49 & $\uparrow$ & 36 & 36 & 36 & 36 & 36.0 & 51.80 \\
\hline 50 & $\uparrow$ & 35 & 35 & 35 & 35 & 35.0 & 53.87 \\
\hline 51 & $\rightarrow$ & 33 & 33 & 33 & 33 & 33.0 & 51.11 \\
\hline 52 & $\rightarrow$ & 33 & 33 & 33 & 33 & 33.0 & 48.10 \\
\hline 53 & $\rightarrow$ & 33 & 33 & 33 & 33 & 33.0 & 48.66 \\
\hline 54 & $\rightarrow$ & 34 & 34 & 34 & 34 & 34.0 & 48.70 \\
\hline 55 & $\rightarrow$ & 34 & 34 & 34 & 34 & 34.0 & 55.28 \\
\hline 56 & $\rightarrow$ & 34 & 34 & 34 & 34 & 34.0 & 55.74 \\
\hline 57 & $\rightarrow$ & 32 & 32 & 32 & 32 & 32.0 & 48.69 \\
\hline 58 & $\rightarrow$ & 34 & 34 & 33.5 & 33.5 & 33.8 & 47.95 \\
\hline 59 & $\rightarrow$ & 34 & 33.5 & 33.5 & 33.5 & 33.6 & 55.79 \\
\hline 60 & $\rightarrow$ & 33 & 33 & 33 & 33 & 33.0 & 54.57 \\
\hline
\end{tabular}




\section{Table 23}

Experimental data obtained for C50 concrete samples - Set 3

\begin{tabular}{|c|c|c|c|c|c|c|c|}
\hline Item & Position & 11 & 12 & 13 & 14 & la & $f_{c}(M P a)$ \\
\hline 61 & $\downarrow$ & 31 & 31 & 31 & 31 & 31.0 & 49.20 \\
\hline 62 & $\downarrow$ & 31 & 31 & 31 & 31 & 31.0 & 49.50 \\
\hline 63 & $\downarrow$ & 32 & 32 & 32 & 32 & 32.0 & 47.35 \\
\hline 64 & $\downarrow$ & 32 & 32 & 32 & 32 & 32.0 & 50.87 \\
\hline 65 & $\downarrow$ & 32 & 32 & 32 & 32 & 32.0 & 47.64 \\
\hline 66 & $\downarrow$ & 32 & 32 & 32 & 32 & 32.0 & 48.41 \\
\hline 67 & $\downarrow$ & 34 & 34 & 32 & 32 & 33.0 & 51.86 \\
\hline 68 & $\downarrow$ & 34 & 34 & 34 & 34 & 34.0 & 49.40 \\
\hline 69 & $\downarrow$ & 34 & 32 & 34 & 32 & 33.0 & 50.67 \\
\hline 70 & $\downarrow$ & 33 & 33 & 33 & 33 & 33.0 & 47.47 \\
\hline 71 & $\uparrow$ & 36 & 36 & 34 & 34 & 35.0 & 49.07 \\
\hline 72 & $\uparrow$ & 36 & 36 & 36 & 36 & 36.0 & 47.52 \\
\hline 73 & $\uparrow$ & 35 & 35 & 35 & 35 & 35.0 & 50.23 \\
\hline 74 & $\uparrow$ & 36 & 36 & 36 & 36 & 36.0 & 51.32 \\
\hline 75 & $\uparrow$ & 36 & 36 & 36 & 36 & 36.0 & 54.05 \\
\hline 76 & $\uparrow$ & 37 & 37 & 37 & $22 * *$ & 37.0 & 52.13 \\
\hline 77 & $\uparrow$ & 34 & 34 & 34 & 34 & 34.0 & 51.34 \\
\hline 78 & $\uparrow$ & 33 & 33 & 33 & 33 & 33.0 & 49.10 \\
\hline 79 & $\uparrow$ & 35 & 35 & 35 & 35 & 35.0 & 51.26 \\
\hline 80 & $\uparrow$ & 36 & 34 & 36 & 34 & 35.0 & 56.99 \\
\hline 81 & $\rightarrow$ & 33 & 33 & 33 & 33 & 33.0 & 49.82 \\
\hline 82 & $\rightarrow$ & 33 & 33 & 33 & 33 & 33.0 & 57.92 \\
\hline 83 & $\rightarrow$ & 33 & 33 & 34 & 34 & 33.5 & 53.54 \\
\hline 84 & $\rightarrow$ & 33 & 33 & 31 & 31 & 32.0 & 46.01 \\
\hline 85 & $\rightarrow$ & 31 & 31 & 31 & 31 & 31.0 & 46.00 \\
\hline 86 & $\rightarrow$ & 34 & 34 & 34 & 34 & 34.0 & 47.67 \\
\hline 87 & $\rightarrow$ & 34 & 34 & 34 & 34 & 34.0 & 47.67 \\
\hline 88 & $\rightarrow$ & 33 & 33 & 33 & 33 & 33.0 & 50.51 \\
\hline 89 & $\rightarrow$ & 34 & 32 & 34 & 32 & 33.0 & 50.10 \\
\hline 90 & $\rightarrow$ & 33 & 33 & 32 & 32 & 32.5 & 47.26 \\
\hline
\end{tabular}




\section{Figure 5}

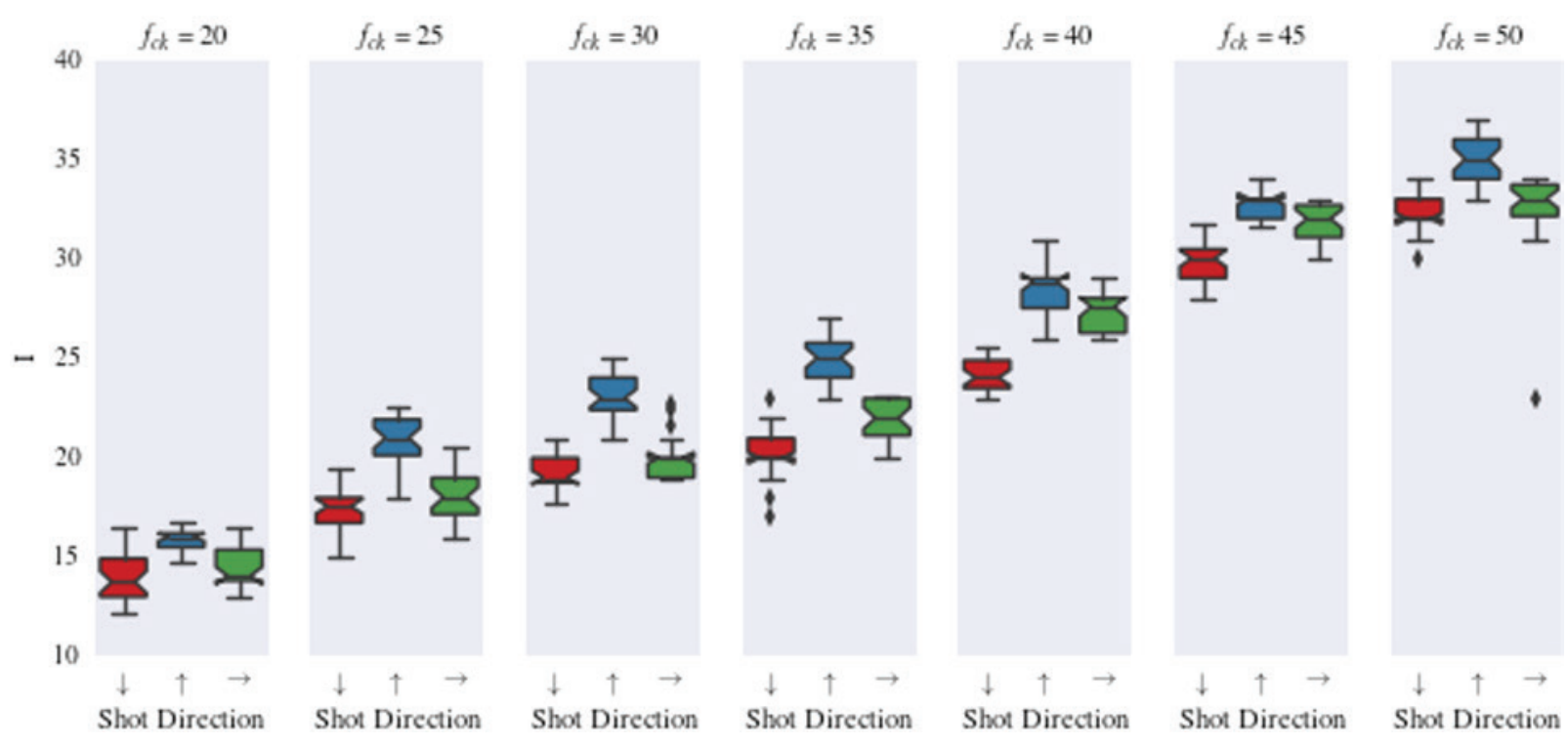

Boxplots for the 3 sets of points grouped according to the shot position

\section{Table 24}

Coefficients of the linear regression models on the form $f_{c}=a l+b$ as a function of each shot position

\begin{tabular}{|c|c|c|c|c|c|}
\hline Position & $\mathbf{a}$ & $\mathbf{b}$ & MSE & R & P \\
\hline $2 \downarrow$ & 1.6045 & -1.4504 & 0.0335 & 0.968 & 0 \\
\hline $1 \rightarrow$ & 1.5403 & -2.1899 & 0.0305 & 0.973 & 0 \\
\hline $3 \uparrow$ & 1.5887 & -6.1778 & 0.0299 & 0.976 & 0 \\
\hline
\end{tabular}

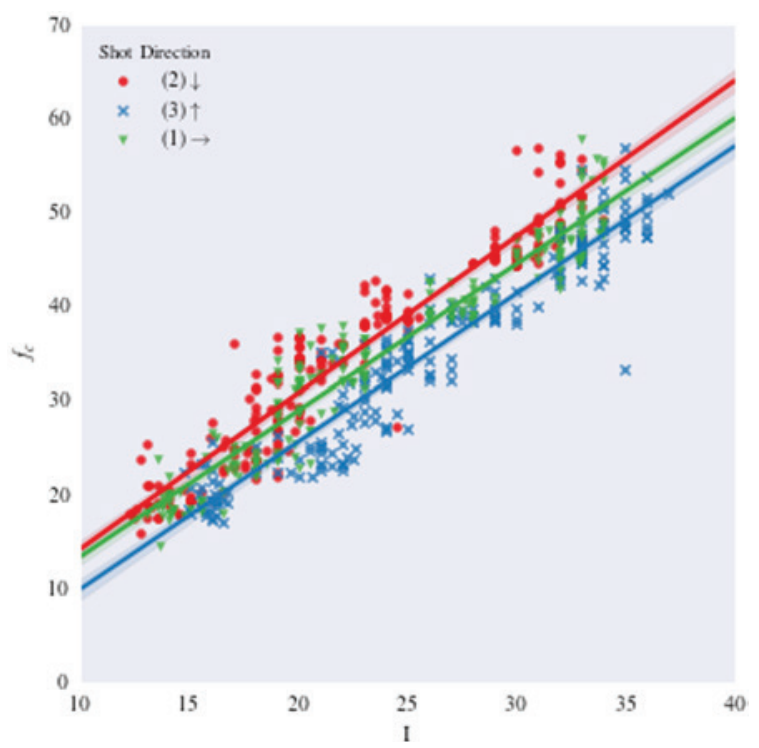

Figure 6

Comparison of the linear regression models adopted for each shot position

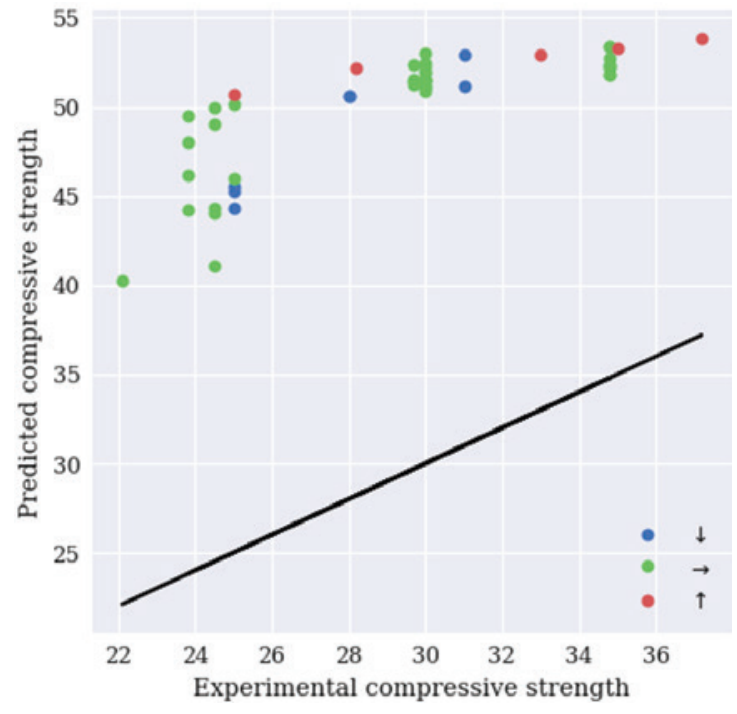

Figure 7

Comparison between values obtained via sclerometer $\left(f_{c s}\right)$ and mechanical tests $\left(f_{c}\right)$ obtained in a construction site 
Figure 8 shows the Two-sided Kolmogorov-Smirnov test (KS-test) for all samples. Each entry shows the p-value for the KS-test, where $p$-values leastest than 0.05 indicates that the samples are drawn from the same continuous distribution. The Two-sided KolmogorovSmirnov tests whether two data samples come from the same distribution. The KS-test has the advantage of making no assumption about the distribution of data (nonparametric and distribution free). The null hypothesis is the two samples come from a common distribution and it is rejected when the associated p-values are greater than 0.05 . A total of 210 pairs were compared, and according to the tests, five pairs of samples were drawn from the same distribution: $(\mathrm{C} 25 \rightarrow 1$ $\mathrm{C} 25 \downarrow),(\mathrm{C} 25 \uparrow / \mathrm{C} 35 \downarrow),(\mathrm{C} 30 \rightarrow / \mathrm{C} 35 \downarrow),(\mathrm{C} 45 \rightarrow / \mathrm{C} 50 \downarrow), \quad(\mathrm{C} 45 \uparrow / \mathrm{C} 50 \rightarrow)$, and the results involving these pairs of data sets shall be carefully analyzed considering the statistical interplay between the groups.

\subsection{Evaluation of concrete structural members}

Based on the data analysis (Botelho et al [11]), an in situ experiment was accomplished on concrete members during construction. The methodology consisted of comparing the fc evaluated via sclerometer measurements, employing the correlation curves proposed in this study. with the fc values obtained from mechanical tests on concrete specimens molded for technological control. The following steps were performed:

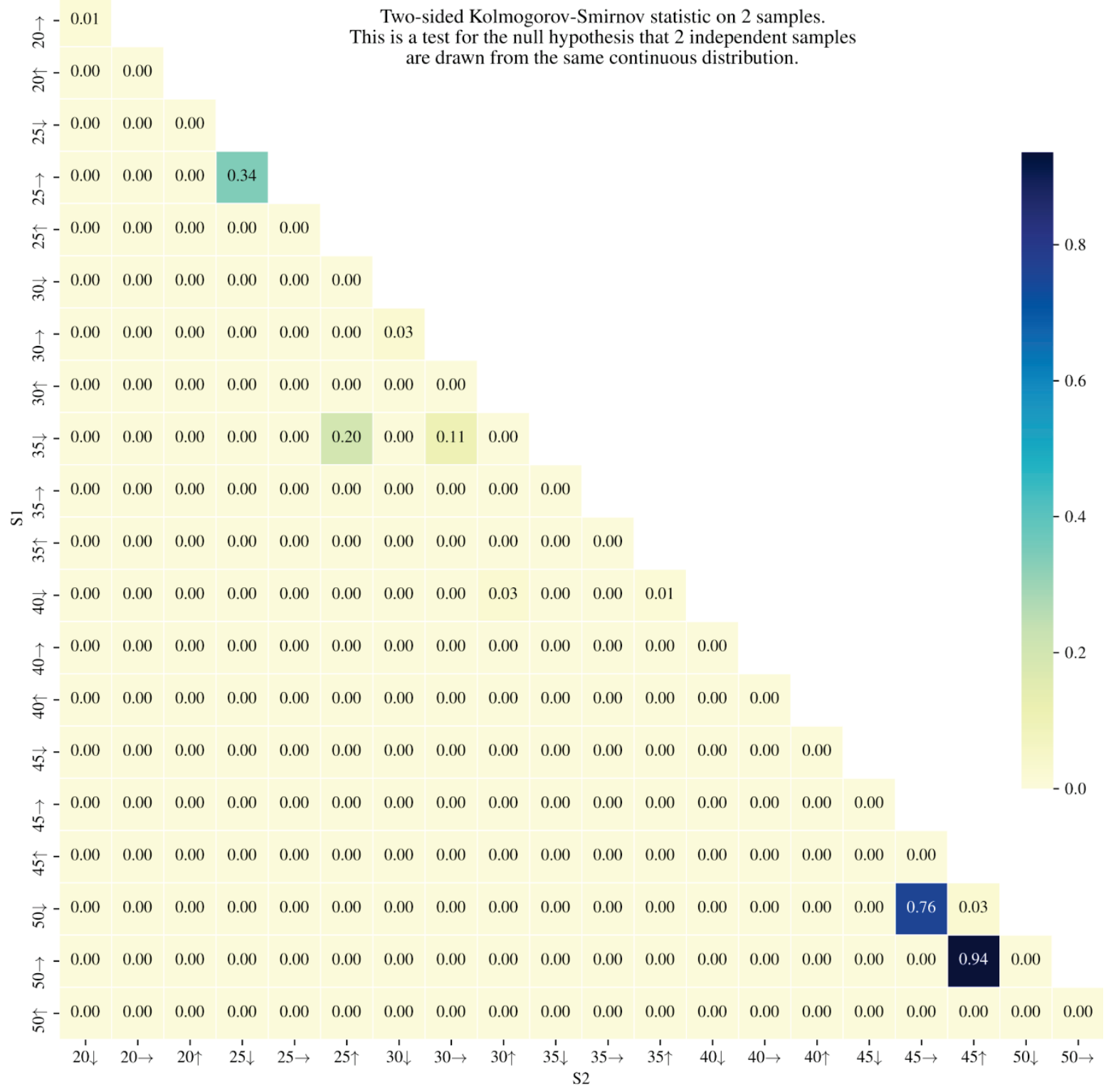

\section{Figure 8}

Two-sided Komolgorov-Smirnov test (KS-test) for all samples. Each entry shows the p-values for the KS-test, where p-values leastest than 0.05 indicates that the samples are dram from the same continuous distribution 
- 48 in situ measurements via sclerometer on concrete elements (see Table 4) according to the employed shot direction: (columns, beams, slabs and foundation blocks) were performed 28 days after casting;

Position $1(\rightarrow): f_{c s}=1.5403$ Ia -2.1899

- the corresponding compressive strength $f_{c s}$ was evaluated via Equations [1], [2] or [3]. employing the $a$ and $b$ coefficients

Position $2(\downarrow): f_{c s}=1.6045 \mathrm{Ia}-1.4504$

\section{Table 25}

Comparison between sclerometer and mechanical tests performed in a construction site

\begin{tabular}{|c|c|c|c|c|c|c|}
\hline Item & Structural member & Shot direction & $\mathbf{I}$ & fc s (MPa) & fc (MPa) & fc / fc s \\
\hline 1 & column & $\rightarrow$ & 35.08 & 51.84 & 34.80 & 1.49 \\
\hline 2 & column & $\rightarrow$ & 36.06 & 53.35 & 34.80 & 1.53 \\
\hline 3 & column & $\rightarrow$ & 35.38 & 52.31 & 34.80 & 1.50 \\
\hline 4 & column & $\rightarrow$ & 34.82 & 51.44 & 29.70 & 1.73 \\
\hline 5 & column & $\rightarrow$ & 34.70 & 51.26 & 29.70 & 1.73 \\
\hline 6 & column & $\rightarrow$ & 33.99 & 50.16 & 25.00 & 2.01 \\
\hline 7 & column & $\rightarrow$ & 34.88 & 51.54 & 29.70 & 1.74 \\
\hline 8 & column & $\rightarrow$ & 35.44 & 52.40 & 29.70 & 1.76 \\
\hline 9 & column & $\rightarrow$ & 34.86 & 51.50 & 30.00 & 1.72 \\
\hline 10 & column & $\rightarrow$ & 34.75 & 51.34 & 30.00 & 1.71 \\
\hline 11 & slab & $\uparrow$ & 37.20 & 52.92 & 33.00 & 1.60 \\
\hline 12 & slab & $\uparrow$ & 35.79 & 50.68 & 25.00 & 2.03 \\
\hline 13 & slab & $\uparrow$ & 36.76 & 52.22 & 28.20 & 1.85 \\
\hline 14 & slab & $\uparrow$ & 37.42 & 53.27 & 35.00 & 1.52 \\
\hline 15 & slab & $\uparrow$ & 37.76 & 53.81 & 37.20 & 1.45 \\
\hline 16 & column & $\rightarrow$ & 35.12 & 51.91 & 30.00 & 1.73 \\
\hline 17 & column & $\rightarrow$ & 34.90 & 51.57 & 30.00 & 1.72 \\
\hline 18 & column & $\rightarrow$ & 36.09 & 53.40 & 34.80 & 1.53 \\
\hline 19 & column & $\rightarrow$ & 35.03 & 51.77 & 34.80 & 1.49 \\
\hline 20 & column & $\rightarrow$ & 35.81 & 52.97 & 30.00 & 1.77 \\
\hline 21 & column & $\rightarrow$ & 34.55 & 51.03 & 30.00 & 1.70 \\
\hline 22 & column & $\rightarrow$ & 34.45 & 50.87 & 30.00 & 1.70 \\
\hline 23 & column & $\rightarrow$ & 35.31 & 52.20 & 34.80 & 1.50 \\
\hline 24 & column & $\rightarrow$ & 35.63 & 52.69 & 34.80 & 1.51 \\
\hline 25 & column & $\rightarrow$ & 35.41 & 52.35 & 34.80 & 1.50 \\
\hline 26 & column & $\rightarrow$ & 35.48 & 52.46 & 30.00 & 1.75 \\
\hline 27 & column & $\rightarrow$ & 35.18 & 52.00 & 30.00 & 1.73 \\
\hline 28 & column & $\rightarrow$ & 35.34 & 52.24 & 30.00 & 1.74 \\
\hline 29 & column & $\rightarrow$ & 34.85 & 51.49 & 30.00 & 1.72 \\
\hline 30 & column & $\rightarrow$ & 27.56 & 40.26 & 22.10 & 1.82 \\
\hline 31 & column & $\rightarrow$ & 28.07 & 41.05 & 24.50 & 1.68 \\
\hline 32 & column & $\rightarrow$ & 33.88 & 50.00 & 24.50 & 2.04 \\
\hline 33 & column & $\rightarrow$ & 30.11 & 44.19 & 23.80 & 1.86 \\
\hline 34 & column & $\rightarrow$ & 31.41 & 46.19 & 23.80 & 1.94 \\
\hline 35 & column & $\rightarrow$ & 30.02 & 44.05 & 24.50 & 1.80 \\
\hline 36 & column & $\rightarrow$ & 32.60 & 48.02 & 23.80 & 2.02 \\
\hline 37 & beam & $\rightarrow$ & 33.55 & 49.49 & 23.80 & 2.08 \\
\hline 38 & beam & $\rightarrow$ & 30.19 & 44.31 & 24.50 & 1.81 \\
\hline 39 & beam & $\rightarrow$ & 33.25 & 49.03 & 24.50 & 2.00 \\
\hline 40 & beam & $\rightarrow$ & 31.25 & 45.94 & 25.00 & 1.84 \\
\hline 41 & beam & $\rightarrow$ & 34.61 & 51.12 & 30.00 & 1.70 \\
\hline 42 & block & $\downarrow$ & 29.29 & 45.55 & 25.00 & 1.52 \\
\hline 43 & block & $\downarrow$ & 28.54 & 44.34 & 25.00 & 1.77 \\
\hline 44 & block & $\downarrow$ & 29.10 & 45.24 & 25.00 & 1.81 \\
\hline 45 & slab* & $\downarrow$ & 32.42 & 50.57 & 28.00 & 2.02 \\
\hline 46 & slab* & $\downarrow$ & 32.80 & 51.18 & 31.00 & 1.83 \\
\hline 47 & slab* & $\downarrow$ & 33.86 & 52.88 & 31.00 & 1.71 \\
\hline 48 & slab* & $\downarrow$ & 32.46 & 50.63 & 28.00 & 1.63 \\
\hline
\end{tabular}


Position $3(\uparrow): f_{c s}=1.5887$ Ia -6.1778

where la is the average for 16 sclerometer readings, as specified by codes.

- $f_{c s}$ values were compared with fc obtained from 28 days old cylindrical concrete samples, cast for technological control purposes.

\subsection{Results}

In spite of the high $\mathrm{R}$ values of the three adopted correlation curves, given by Equations 1-3, which denote a quite good correlation with the experimental database, in situ measurements generated rather discrepant values for the compressive strength of the material in study. As one can see in Table $25, \mathrm{f}_{\mathrm{cs}} / \mathrm{f}_{\mathrm{c}}$ ratios range from $45 \%$ to $108 \%$. This pattern may be clearly observed in Figure 7, where $f_{c} \times f_{c s}$ points are plotted along with a bisector line. An aspect that deserves attention is the fact that the adopted correlation curves are against safety, by taking the $f_{c}$ values measured on concrete samples via mechanical tests as a reference.

Table 26 shows the correlation coefficients for the models presented in this paper. The results for slab (vertical shot position) member present higher correlation values whereas a moderated correlation can be observed for slab (downward position) and for column members. Poor correlations were obtained for beam

Table 26

Correlation coefficients for each member and position. The number of samples is shown in the third column

\begin{tabular}{|c|c|c|c|}
\hline Position & Member & N $^{\circ}$ samples & Corr. coefficient \\
\hline$\rightarrow$ & beam & 5 & 0.543865 \\
\hline$\downarrow$ & block & 3 & - \\
\hline$\rightarrow$ & column & 31 & 0.789565 \\
\hline$\uparrow$ & slab & 5 & 0.971064 \\
\hline
\end{tabular}

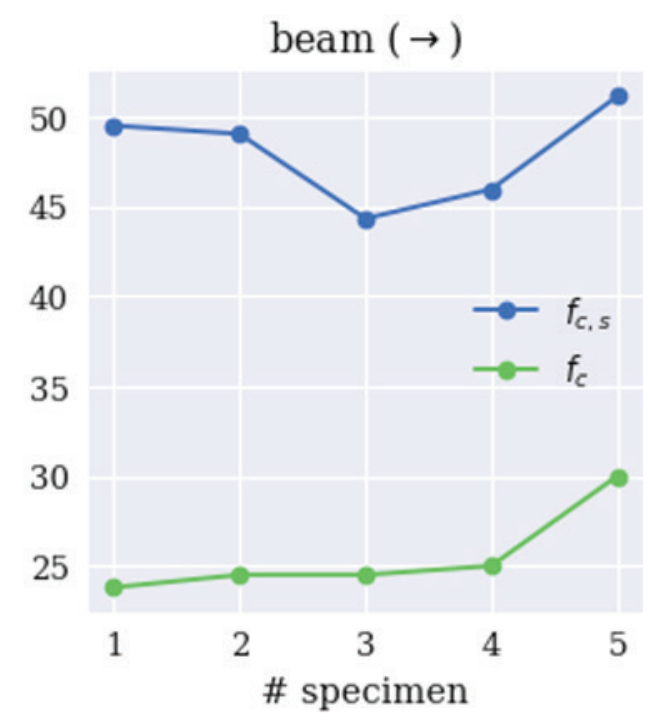

$\operatorname{slab}(\uparrow)$

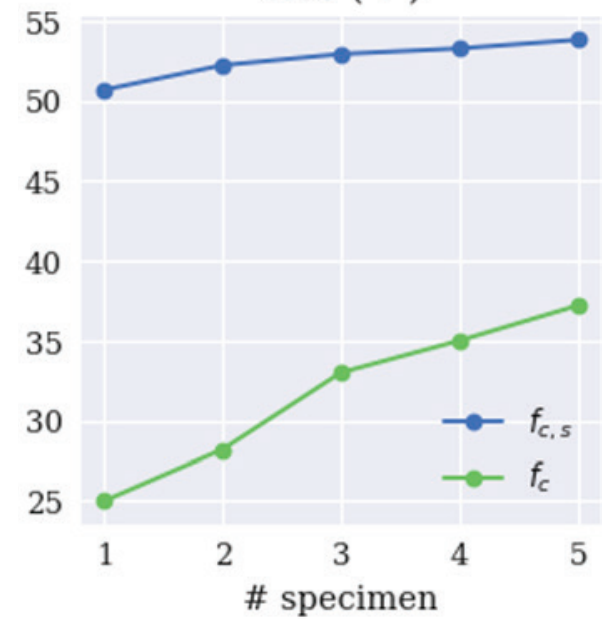

block $(\downarrow)$

45

40

35

$\longrightarrow f_{c, s}$

$\longrightarrow f_{c}$

30

25

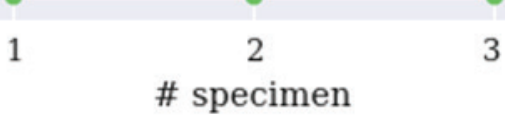

slab* $(\downarrow)$

50

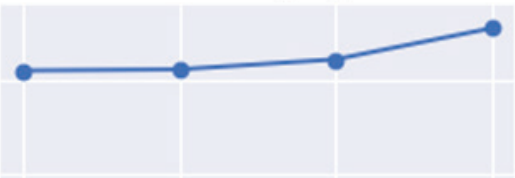

45

40

$\longrightarrow f_{c, s}$

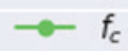

35

30

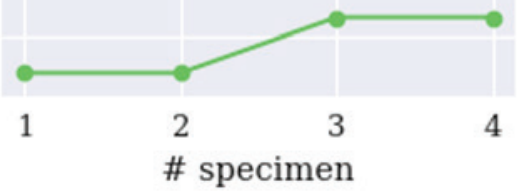

\section{Figure 9}

Compressive strength values: experimental $\left(f_{c}\right)$ and estimated $\left(f_{c s}\right)$ according to shot direction for structural members beam, block and slab 


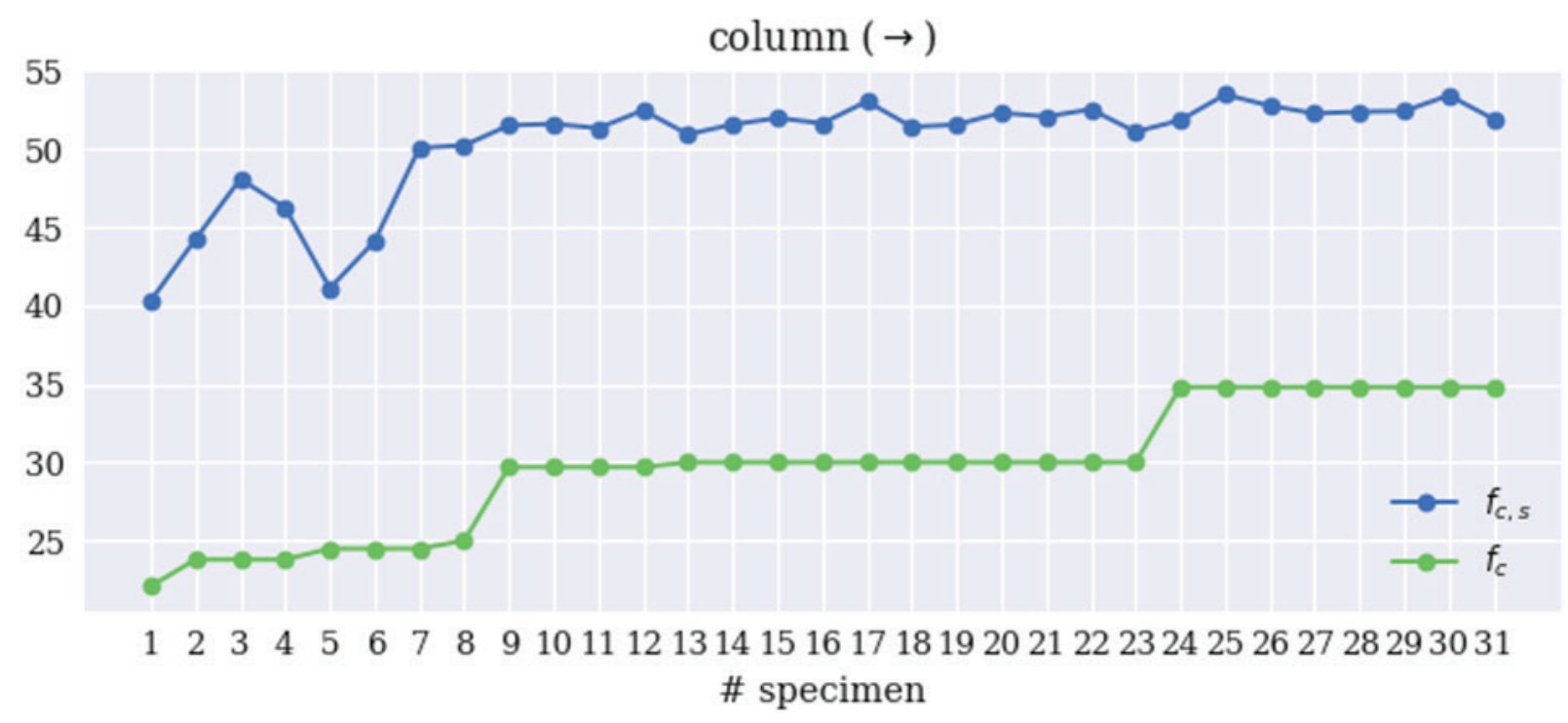

Figure 10

Compressive strength values: experimental $\left(f_{c}\right)$ and estimated $\left(f_{c s}\right)$ according to shot direction for structural member column

member in the horizontal shot position. For the block member, it was not allowed to calculate the correlations due to the small number of samples available. One can observe moderate and higher correlation coefficients for some members, however a proper analysis involves considering the error when using the expressions given in Equations 1, 2 and 3.

Figures 9 and 10 show the experimental $\left(f_{c}\right)$ and estimated $\left(f_{c s}\right)$ compressive strength values according to shot direction for structural members beam, block, column and slab. The estimated values were obtained using Equations 1,2 and 3 depending on the position of the sclerometer. Percentage dif-

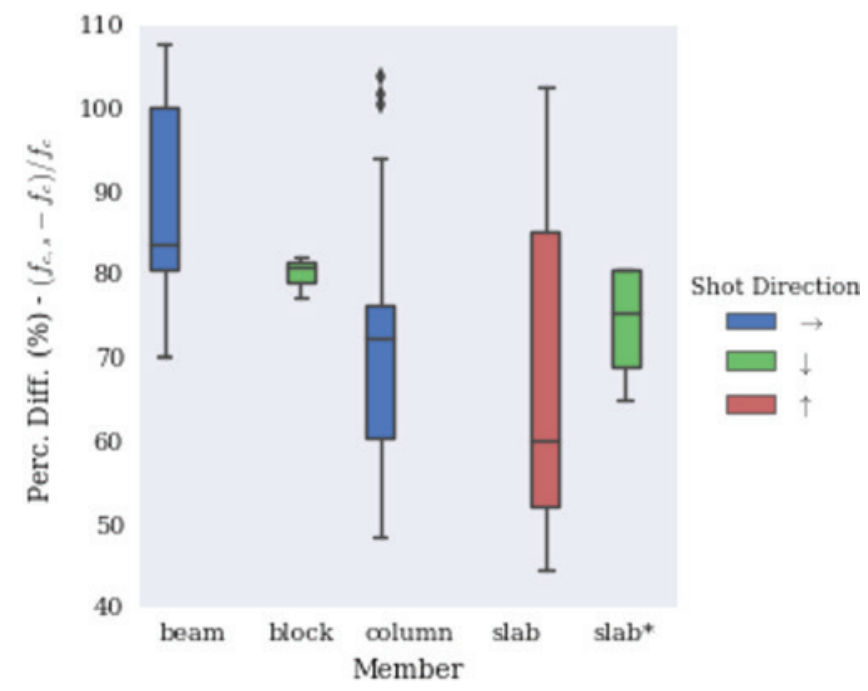

Figure 11

Percentage difference between experimental $\left(f_{c}\right)$ and estimated $\left(f_{c s}\right)$ compressive strength according to shot direction for structural member ferences between experimental $\left(f_{c}\right)$ and estimated $\left(f_{c s}\right)$ compressive strength are shown in Figure 11. As can be seen in this figure, there are an overestimation of the compressive strength which in extreme cases may reach $100 \%$. Several factors such as humidity, carbonatization, chemical aggressions, microcracks and history of the concrete influence the sclerometric index value. The concrete is permeable and the carbon dioxide may react with the substances in the concrete leading to the phenomenon of carbonatization. Cracking allows the penetration of water and carbon dioxide oxidating the armature. An interesting study is establishing a correction factor of the readings obtained take into consideration the carbonatization phenomenon by comparing the sclerometric test results conducted on carbonatated and non-carbonatated specimens. The analysis conducted in this paper denotes the need for more careful evaluation of the protocols concerning the application of sclerometry on real life situations. The authors consider that it is paramount to establish a careful methodology for obtaining adequate correlation curves, as well as the definition of calibration procedures aiming the generalization of the technic to the wide variety of concretes and scenarios in the construction industry.

\section{Conclusions}

In spite of the fact that the application of sclerometry is increasing all over the world, due to its feasibility and non-destructive characteristics, it is paramount to establish protocols that allow adequate correlation curves. The experimental results presented herein denote the necessity of specific codes for the safe application of this technique in practical applications. To this end, it is necessary to perform further experimental investigations. This study is a preliminary step to a broader research aiming to contribute to improvements of the Brazilian code NBR 7584:2012 (ABNT [8]). 


\section{Acknowledgements}

The authors acknowledge the financial support from: Fundação de Amparo à Pesquisa de Minas Gerais - FAPEMIG. Conselho Nacional de Desenvolvimento Científico e Tecnológico - CNPq. Coordenação de Aperfeiçoamento de Pessoal de Nível Superior - CAPES and Pró-Reitoria de Pós-Graduação e Pesquisa da Universidade Federal de Juiz de Fora - PROPP/UFJF.

\section{References}

[1] ABNT - ASSOCIAÇÃO BRASILEIRA DE NORMAS TÉCNICAS. Agregados - Determinação da massa unitária e do volume de vazios. NBR NM 45. Rio de Janeiro. 2006.

[2] _ Agregados - Determinação da composição granulométrica. NBR NM 248. Rio de Janeiro. 2003.

[3] __ Agregados - Determinação do material fino que passa através da peneira 75 um. por lavagem. NBR NM 46. Rio de Janeiro. 2003.

[4] __ Agregado miúdo - Determinação da massa específica e massa específica aparente. NBR NM 52. Rio de Janeiro. 2009.

[5] _ Agregado graúdo - Determinação da massa específica. massa específica aparente e absorção de água. NBR NM 53. Rio de Janeiro. 2009.

[6] Concreto - Procedimento para moldagem e cura de corpos de prova. NBR 5738. Rio de Janeiro. 2015.

[7] ___ Concreto - Ensaios de compressão de corpos de prova cilíndricos. NBR 5739. Rio de Janeiro. 2007.

[8] Concreto endurecido - avaliação da dureza superficial pelo esclerômetro de reflexão. NBR 7584. Rio de Janeiro. 2012.

[9] ALWASH. M.. BREYSSE. D.. SBARTAI. Z.. SZILÁGYI. K. BOROSNYÓl. A.. Factors affecting the reliability os assessing the concrete strength by rebound hammer and cores. Construction and Building Materials (140) 354-363. 2017.

[10] AYMAN. M.. MOHAMED. A.. E.. Mechanical properties of high strength concrete with scrap tire rubber. Construction and Building Materials (93) 249-256. 2015.

[11] BOTELHO. I. T.. DIAS. J.P.. SILVA. G. A. F.. FONSECA. L.G.. FARAGE. M.C.R.. OLIVEIRA. T.M.. BONIFACIO. A.L.. Relationship between the sclerometric index and the compressive strength of concretes. Sodebras (12-141). 2017.

[12] BREYSSE. D.. KLYS. G.. DÉROBERT. X.. SIRIEIX. C.. LATASTE. J.F.. How to combine several non-destructive techniques for a better assessment of concrete structures. Cement and Concrete Research. (38-6). 783-793. 2008.

[13] FAN. X.; HU. S.; LU. S.; WEI. C.. Acoustic emission properties of concrete on cynamic tensile test. Construction and Building Materials. (114) 66-75. 2016.

[14] FORT. R.; BUERGO. M. A.; PEREZ-MONTSERRAT. E. M.. Non-destructive testing for the assessment of granite decay in heritage structures compared to quarry stone. International Journal of Rock Mechanics and Mining Sciences. (61) 296-305. 2013.

[15] FIGUEIREDO FILHO. D. B.. SILVA JUNIOR. J. A.. Desvendando os mistérios do coeficiente de correlação de Pearson (r). Revista Política Hoje. (19-1) (in Portuguese).
[16] MALHOTRA. V.M.; CARINO. N.J.. Handbook on Nondestructive Testing Of Concrete. 2ed. CRC Press. Boston: 2004.

[17] MEHTA. P. K.; MONTEIRO. P. J. M.. Concreto: microestrutura. propriedades e materiais. 2ed. Ibracon. São Paulo: 2014.

[18] SARDAR. K. U. R.; SAINAH. I.; SAHZIM; A. M.; MOHAMMED. J.. Nondestructive test methods for concrete bridges: A review. Construction and Building Materials. (107) 58-86; 2016. 